\title{
Slip of a Two-degree-of-freedom Spring-slider Model in the Presence of Slip-dependent Friction and Viscosity
}

\author{
Jeen-Hwa Wang ${ }^{1}$ \\ ${ }^{1}$ Institute of Earth Sciences, Academia Sinica, Nangang, Taipei, Taiwan
}

\author{
Article history \\ Received October 21, 2016; accepted February 26, 2017. \\ Subject classification: \\ Two-degree-of-freedom spring-slider model, Slip-dependent friction, Viscosity, Stiffness ratio, Phase portrait.
}

\begin{abstract}
In this study, we study the slip of a two-degree-of-freedom spring-slider model, consisting of two sliders, in the presence of slip-dependent friction due to thermal pressurization and viscosity. Simulation results show that seismic coupling between the two sliders is weak when the stiffness ratio, $s$, of the model is smaller than 5 and/or either $U_{c 1}$ or $U_{c 2}$, which are the characteristic displacements of friction law at the two sliders, is smaller than 0.5. The patterns of motions of two sliders yielded by large $U_{c 1}$ and small $U_{c 2}$ are opposite to those by small $U_{c 1}$ and large Uc2. The ratio, $\varphi$, of static friction force at slider 2 to that at slider 1 is a factor in influencing the motions of two sliders. Higher $\varphi$ leads to a longer delay time to trigger the motion of slider 2 . Slider 2 cannot move when $\varphi$ is higher than a critical value which depends on other model parameters. The presence of viscosity between the sliders and moving plate results in increases in duration times and predominant periods of motions of sliders and depresses the generation of an attractor. Viscosity results in small amplitudes and low velocities of motions of sliders.
\end{abstract}

\section{Introduction}

The rupture processes of an earthquake essentially consist of three steps: nucleation (or initiation), dynamical propagation, and arrest. It is necessary to study the mechanisms controlling the whole rupture processes. Such processes are very complicated and can be controlled by several factors. The major factors include brittle-ductile fracture rheology [Jeffreys, 1942; Scholz, 1990], pore fluid pressure [Scholz, 1990], and thermal pressurization [Rice, 2006].

Friction is one of the most important factors in controlling earthquake dynamics [Nur, 1978; Dieterich, 1979; Ruina, 1983; Knopoff et al., 1992; Rice, 1993; Wang, 1996, 1997, 2000, 2012; Rubin and Ampuero, 2005; Ampuero and Rubin, 2008; Bhattacharya and
Rubin, 2014]. The friction coefficient, $\mu(=0.6-0.8$ in general), is defined as the ratio of shear stress, $\tau$, to the effective normal stress, $\sigma_{\text {eff }}$, on the fault plane [Byerlee, 1978]. The frictional force between two contact planes is classically considered to drop from static one to dynamic one after the two planes move relatively. Indeed, the friction law that has been inferred from laboratory experiments is quite complicated and not completely understood, especially for that on natural faults due to limited observational constraints. This makes the proper constitutive law for fault friction an elusive mathematical formulation. The commonly-used friction law is the rate- and state-dependent friction law with the state evolution laws [Dieterich, 1972, 1979; Ruina, 1983; Shimamoto 1986]. A detailed description of the law can be found in several articles [e.g., Marone. 1998; Wang, 2002; Bizzarri and Cocco, 2006c; and Bizzari, 2011c].

Several simple friction laws have been taken into account by some researchers, for examples, a velocity-dependent, weakening-hardening friction law [Burridge and Knopoff, 1967]; a purely nonlinearly velocity-weakening friction law [Carlson and Langer, 1989]; a piecewise, linearly velocity-dependent weakening-hardening friction law [Wang, 1995, 1996, 2012]; a nonlinearly velocity-weakening friction law [Noda et al., 2009]; a displacement softening-hardening friction law [Cao and Aki, 1984/85]; and a piecewise, linearly slip-dependent friction law [Ionescu and Campillo, 1999; and Urata et al., 2008]. Cochard and Madariaga [1994] and Madariaga and Cochard [1994] assumed that purely velocity-dependent friction models can lead to unphysical phenomena or mathematically ill-posed problems. This means that the purely velocity-dependent friction 
law is very unstable at low velocities both during the passage of the rupture front and during the possible slip arrest phase. Ohnaka [2003] stressed that purely velocity-dependent friction is in contrast with laboratory evidence. On the other hand, Lu et al. [2010] assumed that velocity-weakening friction plays an important role on earthquake ruptures. Bizzarri [2011c] deeply discussed this problem.

From theoretical studies, Bizzarri and Cocco [2006a,b,c] revealed that melting of rocks and fault gouge is likely to occur even with the inclusion of the thermal pressurization of pore fluids. Moreover, the dramatic fault weakening at high slip rates predicted by the flash heating of micro-asperity contacts is not able to avert melting [Bizzarri, 2009]. When fluids are present in faults, thermal pressurization can play a significant role on earthquake rupture and also result in resistance on the fault plane [Sibson, 1973; Fialko, 2004; Bizzari and Cocco, 2006a,b,c; Rice, 2006; Wang, 2009, 2011, 2013, 2016a; Bizzarri, 2010; Bizzarri, 2011a,b]. In this study, a slip-weakening friction law induced by thermal pressurization will be taken into account.

Among numerous friction laws, the only constitutive law able to avoid the melting is a slip- and velocity-weakening friction law [Sone and Shimamoto, 2009; Bizzarri, 2010], for which the fault weakening is so dramatic that it cannot be counterbalanced by the resulting enhanced slip velocities. However, both thermal pressurization of pore fluids and flash heating predict not only a very dramatic stress drop, but also a very high peak in fault slip velocity, so that the final result is that melting temperature is very often exceeded, unless the slipping zone is extremely large [Bizzarri and Cocco, 2006b; Bizzarri, 2009]. Bizzarri [2011a] stressed that when melting occurs, the rheological behavior of the fault zone no longer obeys the Coulomb-AmontonMohr formulation, in that a viscous rheology is needed to describe the traction evolution during the ruptures.

Jeffreys [1942] first emphasized the importance of viscosity on faulting. Viscosity in a fault can be controlled by frictional melts [Byerlee, 1968]. Temperature, pressure, water content, etc., will influence viscosity [Turcotte and Schubert, 1982]. Scholz [1990] suggested that the residual strength of fault-generated friction melts would be high and so present significant viscous resistance to shear. This inhibits continued slip. Spray [1993; 1995] observed that most pseudotachylytes [Sibson, 1975] are partial melts possessing low viscosity, and capable of generating a sufficient melt volume to reduce the effective normal stress. Thus, friction melts can act as fault lubricants during co-seismic slip and viscosity decreases with increasing temperature [Spray, 2005]. Rice et al. [2001] discussed the physical basis of rate- and state-dependent friction, including the direct effect in thermally activated processes allowing creep slippage at asperity contacts on the fault surface. Wang [2007] stressed the importance of viscosity on earthquake ruptures. Wang [2011] assumed that quartz plasticity could be formed in the main slip zone of the earthquake when $\mathrm{T}>300{ }^{\circ} \mathrm{C}$ after the fault ruptured. The shear zone with quartz plasticity would be localized in a very thin heated layer, for example, 5-mm thick layer. Quartz plasticity could lubricate the fault plane at higher $\mathrm{T}$ and yield viscous stresses to resist slip at lower T. Some researchers have already investigated the effect of viscosity in springblock system [e.g., Shaw, 1994; Yoshino, 1998; Hainzl et al., 1999; Wang, 2016a]. On the other hand, several researchers [Knopoff et al., 1973; Cohen, 1979; Xu and Knopoff, 1994; Knopoff and Ni, 2001] took the viscous effect as a factor in causing seismic radiation to reduce energy during earthquake ruptures.

Since the ingredients of an ideal model are only partly understood, a set of equations to describe comprehensively fault dynamics has not yet been established. Nevertheless, some models, for instance the crack model and dynamical spring-slider model, have been developed to approach fault dynamics for a long time. Although the frictional effect on earthquake ruptures has been widely studied as mentioned above, the studies of viscous effect on earthquake ruptures are rare. The viscous effect mentioned in Rice et al. [2001] was an implicit factor which is included within the direct effect of rate- and state-dependent friction law. Wang [2016a] studied frictional and viscous effects on slip of a one-degree-of-freedom spring-slider model associated to a fault. However, the interaction between two sliders related to two faults or two fault segments. In this work, I will explore the effects of slip-weakening friction due to thermal pressurization and viscosity on earthquake ruptures based on a two-degree-offreedom spring-slider model, which is generally used to approach an earthquake fault, because numerous earthquakes consist of few segments [Galvanetto, 2002; Turcotte, 1992; Dragoni and Santini, 2010, 2011, 2012, 2014; and cited references herein]. For example, Wang [2007] applied this model to study the difference in ground motions between the northern and southern segments of the Chelungpu fault, along which the 1999 Chi-Chi, Taiwan, $M_{s} 7.6$ earthquake ruptured. In this study, the frictional factors include the characteristic displacement of the friction law and the differ- 
ence on static friction forces between two sliders. The viscous effect is represented by an explicit parameter. Included also is the seismic coupling which will be defined below. The effects on the possibility of simultaneous motions of two sliders, which will lead to a larger-sized event, due to seismic coupling and friction will be studied. The frictional and viscous effects on interaction between two sliders, including the triggering of the second slider by the first one, will be studied. Results will be significant on the understanding of earthquake ruptures.

\section{Two-degree-of-freedom Spring-slider Model}

\subsection{Model}

The model consists of two sliders of mass $m_{i}$ $(i=1,2)$ and three spring. One coil spring of strength $\mathrm{K}$ links two sliders and each slider is also pulled by a leaf spring of strength $L_{i}(i=1,2)$ from a moving plate with a constant velocity $\mathrm{v}_{\mathrm{P}}$ (Figure 2 ). The moving plate provides the driving force on the sliders. At time $t=0$, all sliders rest in an equilibrium state. The $\mathrm{i}$-th slider is located at position $u_{i}$, measured with respect to its initial equilibrium position, along the x-axis. Each slider is subjected to a slip- dependent frictional force, $F_{i}\left(u_{i}\right)$ $(i=1,2)$, where $u i$ is the displacement of the $i$-th slider, and a velocity-dependent viscous force, $\Phi\left(v_{i}\right)$, where $v_{i}=d u_{i} / d t$ is the velocity of the $i$-th slider. The equation of motion of the system is: (1a)

$$
\begin{aligned}
& m_{1}\left(d^{2} u_{1} / d t^{2}\right)=K\left(u_{2}-u_{1}\right)-L_{1}\left(u_{1}-v_{p t}\right)-F_{1}\left(u_{1}\right)-\Phi\left(v_{1}\right) \\
& m_{2}\left(d^{2} u_{2} / d t^{2}\right)=K\left(u_{1}-u_{2}\right)-L_{2}\left(u_{2}-v_{p t}\right)-F_{2}\left(u_{2}\right)-\Phi\left(v_{2}\right)
\end{aligned}
$$

It is noted that the total forces in Equation (1) are null when the sliders do not slide, that is, $v_{i}=0$. For simplification, the inertial effect is considered to be equal for the two sliders, i.e., $m_{1}=m_{2}=m$. Considering the two sliders to be two segments of a single earthquake fault, the coupling between the moving plate and each fault segment should be equal, thus giving $L_{1}=L_{2}=L$.

Equation (1) consists of two processes: The first one is the coupling process between the moving plate and a slider through the leaf spring $L$. The other one is the generation of "self-stress" [Andrews, 1978], which originates from the joint effect of the coil spring $K$ between two sliders and the leaf spring $L$. The coil spring $K$ plays a role only in transferring energy from one slider to the other; thus, it does not change the total energy of the system. However, the leaf spring $L$ plays two roles: One is the supply of energy to the system from the driving force caused by the moving plate, i.e., the $L v_{p} t$ term in Equation (1), and the other is the loss of energy from the system. Hence, the leaf spring $L$ can change the total energy in the system. Therefore, the stiffness ratio $s=K / L$ is a significant parameter representing the level of conservation of energy in the system [Wang, 1995]. In this study, s is taken to represent seismic coupling. Larger $s$ shows that the coupling between two sliders is stronger than that between a slider and the moving plate. This results in a smaller loss of energy through the $L$ spring, thus indicating a higher level of conservation of energy in the system. Of course, smaller s indicates a lower level of conservation of energy. When $L$ is constant, small $K$ (less than a critical value) can produce an unstable rupture. When $K<<L$ or $K=0$, Equation (1) becomes:

$$
m d u_{i} / d t=-L\left(u_{i}-v_{p} t\right)-F_{i}\left(u_{i}\right)-\Phi\left(v_{i}\right)(i=1,2)
$$

Obviously, the system loses the coupling between two sliders, and thus each slider moves independently. Hence, the system can only generate a small event consisting of only one single slider. In other words, large $s$ results in simultaneous motions of the two sliders. When $L<<K$ or $L=0$, the effect due to the leaf spring disappears, and the coupling between two sliders dominates the behavior of the system. Based on a simple 2-D anti-plane strain softening model, Stuart [1981] considered the ratio $K_{f} / K_{s}$, where $K_{f}$ and $K_{s}$ are the stiffness of the fault zone and that of the elastic surroundings, respectively, to be a significant indicator of earthquake instability. He stressed that instability occurs when the ratio reaches unity, i.e., $K_{f} \approx K_{s}$. His ratio is similar to $s=K / L$ defined by Wang [1995].

This model displayed by Equation (1) addresses only the strike-slip component and, thus, cannot completely represent earthquake ruptures, which also consist of transpressive components. Nevertheless, simulation results of this model can still reveal significant information on earthquake ruptures. Of course, some reformed spring-slider models, for examples those proposed by He et al. [1998] and Ryabov and Ito [2001], have been applied to study dip-slip faulting.

\subsection{Viscosity}

For deformed materials, there are two components, i.e., elastic component and viscous component, when the viscous effect is present. The elastic component can be modeled as a spring with an elastic constant $E$, given by the formula: $\sigma=E \varepsilon$, where $\sigma, E$, and $\varepsilon$ are, respectively, the stress, the elastic modulus of the ma- 


\section{a: Kelvin-Voigt Model b: Maxwell Model}

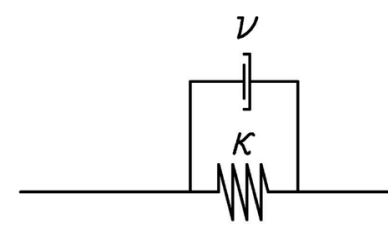

(a)

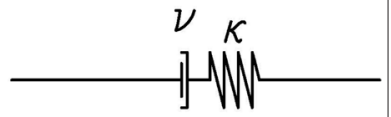

(b)

Figure 1. The two types of viscous materials: (a) for the KelvinVoigt model and (b) for the Maxwell model. ( $\kappa=$ spring constant and $v=$ coefficient of viscosity).

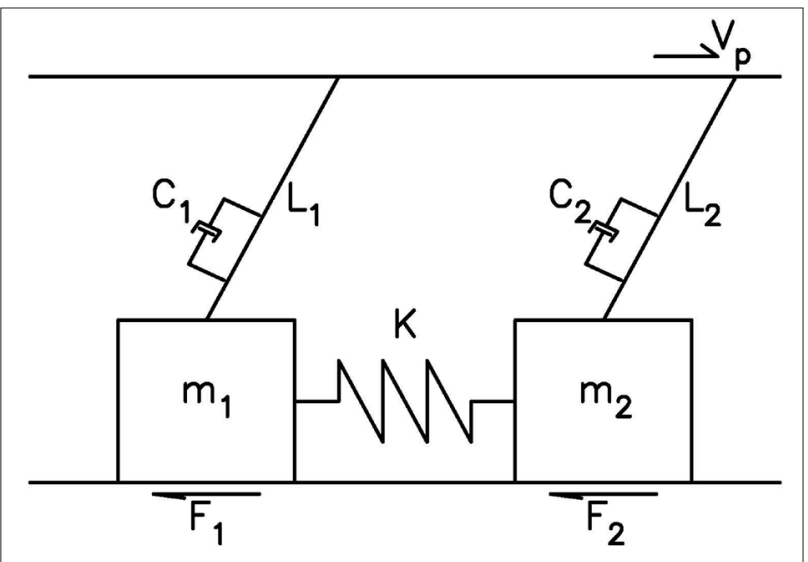

Figure 2. Two-body spring-slider model. In the figure, $m_{i}, K, L_{i}, C_{i}$, $v_{p}$, and $F_{i}$ denote, respectively, the mass, the spring constant between two sliders, the spring constant between a slider and the moving plate, the coefficient of viscosity, the velocity of the moving plate, and the frictional force.

terial, and the strain that occurs under the given stress. The viscous component can be modeled as a dashpot such that the stress-strain rate relationship can be given as, $\sigma=v(d \varepsilon / d t)$ where $v$ is the viscosity of the material. There are two simple and commonly-used models to describe the viscous materials [Jaeger and Cook, 1977; Hudson, 1980]. The first one is the Maxwell model which can be represented by a purely viscous damper and a purely elastic spring connected in series, as shown in Figure 1. The model can be represented by the following equation: $d \varepsilon / d t=d \varepsilon_{D} / d t+d \varepsilon_{S} / d t=\sigma / v+E^{-}$ ${ }^{1} d \sigma / d t$. The model predicts that stress decays exponentially with time. Of course, the behavior of a viscoelastic material depends on boundary conditions. One limitation of this model is that it does not predict creep accurately. The second one is the Kelvin-Voigt model, also known as the Voigt model, consists of a Newtonian damper and Hookean elastic spring connected in parallel, as shown in Figure 1. It is used to explain the creep behavior of materials. The constitutive relation is expressed as: $\sigma(t)=E \varepsilon(t)+v d \varepsilon(t) / d t$. Temperature, pressure, water content, etc., will influence viscosity [Tur- cotte and Schubert, 1982]. Frictional melts can control viscosity in a fault [Byerlee, 1968; Spray, 2005; and cited references herein]. For the Maxwell model, the strain will increase, without a upper limit, with time at constant $\sigma$; while the Kelvin-Voigt model the strain will increases, with an upper limit, with time. Hence, the latter is more appropriate than the former to be applied to the seismological problems. Hence, the Kelvin-Voigt model is taken in this study.

Although viscosity varies with temperature, pressure, and water content, only a constant viscosity for each segment is considered below. The Newtonian viscous force is described by a dash-pot shown in Figure 1 specified with viscosity $v$ between the slider and the moving plate, and, thus, the viscous force at the slider is represented by $-u v$ where $v(=d u / d t)$ is the velocity of the slider. For the Kelvin-Voigt model, the stress is a function of both strain and strain rate and thus can be applied to the seismological problems [Hudson, 1980].

However, it is not easy to directly implement viscosity in a dynamical system as used in this study. Hence, viscosity is here represented in an alternative way. Viscosity leads to the damping of oscillations of a body. The damping coefficient is usually proportional to viscosity and is controlled by the linear dimension of the body in a viscous fluid. For example, according to Stokes' law, the damping coefficient $C$ of a sphere of radius $R$ in a fluid of viscosity $v$ is given by $C=6 \pi R v$ [Kittel et al., 1968]. The damping coefficient is regarded as viscosity hereafter. Hence, the viscous force is represented by $\Phi=C v$. Noted that the unit of $C$ is $\mathrm{N} /(\mathrm{m} / \mathrm{s})$. The model shown in Figure 2 is indeed an approximation to my approach. A complete model should include the viscous effect between two sliders. It would be too complicated to be considered here, because this study is focused only on the viscous effect between a slider and the background moving plate.

\subsection{Friction due to Thermal Pressurization}

As mentioned above, friction can also be produced from thermal pressurization. On a fault plane with an area of $A$ and an average displacement $\overline{\mathrm{u}}$, the frictional energy caused by the dynamic friction stress, $\tau_{d}$, is $E_{f}=\tau_{d} \bar{u} A$ which could result in a temperature rise, $\Delta T$. Frictional heat can conduct outwards from the slipping zone to wall rocks. Theoretical analyses [e.g., Bizzari and Cocco, 2006a,b; Fialko, 2004] show that $\Delta T$ is described by an error function of distance and decays outwards from the fault plane. Under thermal pressurization, the energy and fluid mass conservation equations in a 1-D fault plane, in which the $x$-and y-axes denote 
the directions along and normal to the fault plane, respectively, can be found in Rice [2006].

Rice [2006] proposed two end-members models for thermal pressurization: the adiabatic-undrained-deformation (AUD) model and slip-on-a-plane (SOP) model. The first model corresponds to a homogeneous simple shear strain $\varepsilon$ at a constant normal stress $\sigma_{n}$ on a spatial scale of the sheared layer that is broad enough to effectively preclude heat or fluid transfer. The second model shows that all sliding is on the plane with $\tau(0)=f\left(\sigma_{n}-p_{0}\right)$ where $p_{o}$ is the pore fluid pressure on the sliding plane $(y=0)$. For this second model, heat is transferred outwards from the fault plane. The shear stress -slip functions, $\tau(u)$, caused by thermal pressurization [Rice, 2006] are:

$$
\tau_{\text {aud }}(u)=f\left(\sigma_{n}-p_{o}\right) \exp \left(-u / u_{c}\right)
$$

for the AUD model; and

$$
\tau_{\text {sop }}(u)=f\left(\sigma_{n}-p_{0}\right) \exp \left(u / L^{\star}\right) \operatorname{erfc}\left(u / L^{\star}\right)^{1 / 2}
$$

for the SOP model. The two parameters $u_{c}$ and $L^{\star}$ are the respective characteristic displacements, which are in terms of physical properties of the fault-zone materials. The stress $\tau_{\text {aud }}(u)$ displays exponentially slip-weakening friction. Indeed, The stress $\tau_{\text {sop }}(u)$ also shows slip-weakening [see Wang, 2009]. Since the SOP model is based on a constant velocity, it cannot be used in this study.

For numerical simulations from Equation (1), a slip-weakening friction law: $F(u)=F_{o} \exp \left(-u / u_{c}\right)$ based on the AUD model is taken into account. The variations of friction force versus displacement for five values of $u_{c}$, i.e., 0.1, 0.3, 0.5, 0.7, and $0.9 \mathrm{~m}$, are displayed in Figure 3. The friction force decreases with displacement. Ob-

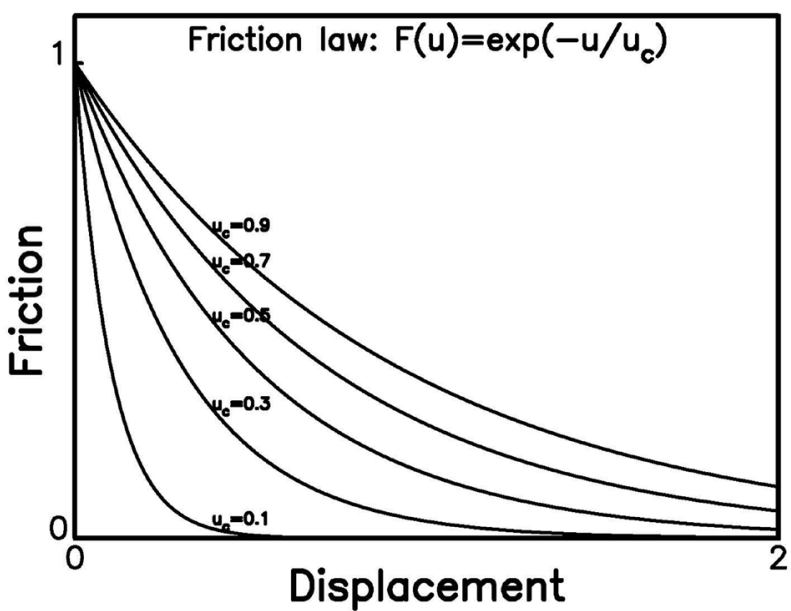

Figure 3. Three slip-weakening friction law: for $F(u)=F_{o} \exp \left(-u / u_{c}\right)$ with $u_{c}=0.1,0.3,0.5,0.7$, and $0.9 \mathrm{~m}$ when $F_{o}=1$ unit. viously, the friction force decreases faster for smaller uc than for larger uc and the force drop decreases with increasing $u_{c}$. For small $u, \exp \left(-u / u_{c}\right)$ can be approximated by $1-u / u_{c}$ [Wang, 2016b]. Obviously, $u_{c}^{-1}$ is almost the decreasing rate, $\gamma$, of friction force with displacement at small $u$. Thus, small (large) $u_{c}$ leads to large (small) $\gamma$.

Substituting the friction law and the viscous law into Equation (1) leads to

$m\left(d^{2} u_{1} / d t^{2}\right)=K\left(u_{2}-u_{1}\right)-L\left(u_{1}-v_{P} t\right)-F_{o 1} \exp \left(-u_{1} / u_{c 1}\right)-C_{1} d u_{1} / d t$

$m\left(d^{2} u_{2} / d t^{2}\right)=K\left(u_{1}-u_{2}\right)-L\left(u_{2}-v_{P} t\right)-F_{o 2} \exp \left(-u_{2} / u c_{2}\right)-C_{2} d u_{2} / d t(5 b)$

To deal with the problem easily, we normalize Equation (5). Letting $F_{o 1}=F_{o}, F_{o 2}=\varphi F_{o}, D_{o}=F_{o} / L$, $\omega_{o}=(L / m)^{1 / 2}, \tau=(L / m)^{1 / 2} t=\omega_{o} t, U_{c 1}=u_{c 1} / D_{o}, U_{c 2}=u_{c 2} /$ $D_{o}, \quad \eta_{1}=C_{1} \omega_{o} / L, \quad \eta_{2}=C_{2} \omega_{o} / L, V_{P}=v_{P} / D_{o} \omega_{o}, U_{i}=u_{i} / D_{o}$, $V_{i}=d U_{i} / d \tau, d u_{i} / d \tau=\left[F_{o} /(m L)^{1 / 2}\right] d U_{i} / d \tau$, and $d^{2} u_{i} / d \tau^{2}=$ $\left(F_{o} / m\right) d^{2} U_{i} / d \tau^{2}$, Equation (5) becomes:

$d^{2} U_{1} / d \tau^{2}=s\left(U_{2}-U_{1}\right)-\left(U_{1}-V_{P} \tau\right)-\exp \left(-U_{1} / U_{c 1}\right)-\eta_{1} d U_{1} / d \tau(6 a)$

$d^{2} U_{2} / d \tau^{2}=s\left(U_{1}-U_{2}\right)-\left(U_{2}-V_{P} \tau\right)-\varphi \exp \left(-U_{2} / U_{c 2}\right)-\eta_{2} d U_{2} / d \tau$

Let $y_{1}=U_{1}, y_{2}=U_{2}, y_{3}=d U_{1} / d \tau$, and $y_{4}=d U_{2} / d \tau$. Equation (6) can be re-written as four first-order differential equations:

$$
\begin{gathered}
d y_{1} / d \tau=y_{3} \\
d y_{2} / d \tau=y_{4} \\
d y_{3} / d \tau=-(s+1) y_{1}+s y_{2}-\exp \left(-y_{1} / U_{c 1}\right)-\eta_{1} y_{3}+V_{P} \tau \\
d y_{4} / d \tau=s y_{1}-(s+1) y_{2}-\varphi \exp \left(-y_{2} / U_{c 2}\right)-\eta_{2} y_{4}+V_{P} \tau
\end{gathered}
$$

Obviously, the solutions of Equation (7) exist within a domain determined by six model parameters, i.e., $s$, $\mathrm{U}_{\mathrm{c1}}, \mathrm{U}_{\mathrm{c} 2}, \varphi, \eta_{1}$, and $\eta_{2}$, in a six-dimensional space. Since it is actually difficult to analytically define the domain, numerical computations will be performed. Equation (7) is computed through the fourth-order Runge-Kutta method [Press et al., 1986]. In the following numerical simulations, the sliders are restricted to move only along the positive direction, that is, $V_{i} \geq 0$ and $U_{i} \geq 0(i=1,2)$. For each case, four diagrams are produced from numerical simulations: the time variation in normalized acceleration, $A / A_{\max }$, the time variation in normalized velocity, $V / V_{\max }$, the time variation in normalized displacement, $U / U_{\max }$, and the phase portrait of $V$ versus $U$.

A phase portrait, denoted by $y=f(x)$, is a plot of a physical quantity versus another of an object in a dy- 
namical system [Thompson and Stewart, 1986]. The intersection point of $f(x)$ and the bisection line, i.e., $y=x$, is called the fixed point, that is, $f(x)=x$. If the function $f(x)$ is continuously differentiable in an open domain near a fixed point $x_{f}$ with $\left|f^{\prime}\left(x_{f}\right)\right|<1$, the fixed point is an attractor. In other words, an attractive fixed point is a fixed point $x_{f}$ of a function $f(x)$ such that for any value of $x$ in the domain that is close enough to $x_{f}$, the iterated function sequences, i.e., $x, f(x), f^{2}(x), f^{3}(x), \ldots$, converges to $x_{f}$ An attractive fixed point is a special case of a wider mathematical concept of attractors. Chaos can be generated at some attractors. The details can be seen in Thompson and Stewart [1986] or other nonlinear literatures. Chaotic motion in a two-degree-of-freedom system have been studied by numerous researches [e.g., Huang and Turcotte, 1990a,b, 1992; de Sousa Viera, 1999; and Abe and Kato, 2013]. However, only the friction law was considered in those studies.

\section{Numerical Simulation}

Before performing numerical simulations, we must consider the realistic values of model parameters. It is difficult to directly evaluate the value of $s$, because it is not easy to measure L. Wang [1995] showed that for a higher-order system with the number of sliders being larger than 100, the value of $s$ which will be appropriate for seismicity simulations ranges from 20 to 120 . As mentioned above, large $s$ more easily produces self-organization in a system and thus there is strong coupling between two sliders than small s. Strong coupling makes the two sliders move almost simultaneously. Hence, in order to view somewhat independent motions of respective sliders we take small $s$. Practical tests from numerical computations show that $s<5$ leads to weak coupling and $s \geq 5$ results in strong coupling. Generally, $v_{P}$ is $\sim 10^{-9} \mathrm{~m} / \mathrm{s}$ and thus $V_{P}$ is $\sim 10^{-9}$ when $D_{o} \omega_{o}$ is an order of magnitude of $1 \mathrm{~m} /$ sec. $D_{o}$ is almost the maximum displacement, umax, on the ruptured fault plane. Since the value of $u_{c}$ is rare, it is not easy to evaluate $U_{c}=u_{c} / D_{o}$. From related data of a mature fault surface at $7 \mathrm{~km}$, Rice [2006] obtained $u_{c}=12 \mathrm{~m}$. Since $u_{\max }$ was not given in Rice [2006], $U_{c}$ cannot be estimated. Wang [2009] inferred the value of uc from given data of the 1999 Chi-Chi, Taiwan, $M_{s} 7.6$ earthquake. His estimated value of $u_{c}$ is between 3.3 for $u_{\max }=10.7 \mathrm{~m}$ and $3.5 \mathrm{~m}$ for $u_{\max }=12.3$ $\mathrm{m}$ at shallow depths of the fault zone. The values of $u_{\text {max }}$ were inferred by different researchers. Hence, the value of $U_{c}$ is about between 0.30 and 0.27 from Wang [2009]. Of course, $U_{c}$ could vary with depth and from area to area. Since $\eta=C \omega_{0} / L$, we have $\eta=4 \pi R v \omega_{0} s / K$.
The viscosity, $v$, of a fault zone depends upon the temperature, pressure, water content, $\mathrm{SiO}_{2}$ content, rock types etc. Spray [1993] showed that the calculated values of $v$ for friction melts are low and decrease with increasing temperature. For examples, the values are about $10^{3}-10^{6} \mathrm{~Pa} s$ at $800^{\circ} \mathrm{C}$ and about $10^{1}-10^{3} \mathrm{~Pa} \mathrm{~s}$ at $1200^{\circ} \mathrm{C}$. In general, $v$ lies in the range $10^{15} \mathrm{~Pa}$ and $10^{2} \mathrm{~Pa} \mathrm{~s}$ when the temperature varies from $500^{\circ} \mathrm{C}$ to $1200^{\circ} \mathrm{C}$. Savage and Lachenbrush [2003] mentioned that for a seismogenic zone with a thickness of $14 \mathrm{~km}$, the viscosity on the bottom is $(0.31-3.12) \times 10^{20} \mathrm{~Pa} \mathrm{~s}$. Obviously, the values for lower crustal materials from Savage and Lachenbrush [2003] are higher than those for upper crustal materials from Spray [1993]. The parameter $R$ is almost the dimension of the deformed volume around a rupture fault. Based on stick-slip behavior and elastic rebound theory of a fault, Turcotte and Schubert [1982] estimated the value of $R$. Their values of $R$ are in the range $750 \mathrm{~m}$ to $7500 \mathrm{~m}$ when the average displacement on a fault is $5 \mathrm{~m}$, the stress drop is $10-100 \mathrm{MPa}$, and the shear modulus is $30 \mathrm{GPa}$. The average value of $K$ for numerous faults is about $4: 6 \times 10^{14} \mathrm{~N} / \mathrm{m}$ [Wang, 2012]. Hence, the value of $\eta$ lies in the range $10^{-8}$ to $10^{6}$ for $v$ of $10^{2}$ to $10^{15} \mathrm{~Pa} \mathrm{~s}$ when $\mathrm{s}<10$ and $\omega_{o}=1 \mathrm{~Hz}$.

Simulation results could be different for various time steps, $\delta \tau$. Practical tests suggest that simulation results show numerical stability when $\delta \tau<0.05$ in the following computations, the time step is taken to be $\delta \tau=0.02$. When $V_{P} \tau=\exp \left(-y_{1} / U_{c 1}\right)$ on slider 1 from Equation (4c), the force exerted from the moving plate is equal to the static friction force. In principle, slider 1 can start to move. In practice, the computation cannot go ahead because all values are zero. In order to kick off slider 1 , an initial force, $\delta F$, is necessary for computations. The value of $\delta F$ can affect computations. A very small value of $\delta F$ cannot enforce slider 1 to move; while a large one will dominate computations. Carlson et al. [1991] studied the effect of $\delta F$ (denoted by $\sigma$ in their article) on computational results. Numerical tests show that $\delta F=10^{-3}$ is appropriate for numerical simulations.

Simulations are made for various values of model parameters. Figures 4-13 displays the time variations in $A / A_{\max }, V / V_{\max }$, and $U / U_{\max }$, and the phase portrait of $V$ versus $U$ with various sets of values of model parameters which are displayed in Table 1. In each diagram, the simulation results for slider 1 and slider 2 are represented, respectively, by a solid line and a dotted line. It is noted that the viscous effect taken into account only from Figure 11 to Figure 13. 


\begin{tabular}{lcccccc}
\hline $\begin{array}{l}\text { Number } \\
\text { of Figure }\end{array}$ & $\mathbf{s}$ & $\eta_{\mathbf{1}}$ & $\eta_{\mathbf{2}}$ & $\varphi$ & $\mathbf{U}_{\mathrm{c} 1}$ & $\mathbf{U}_{\mathrm{c} 2}$ \\
\hline $\mathbf{4}$ & 5 & 0 & 0 & 1 & 0.1 & 0.1 \\
$\mathbf{5}$ & 1 & 0 & 0 & 1 & 0.1 & 0.1 \\
$\mathbf{6}$ & 1 & 0 & 0 & 1 & 0.5 & 0.5 \\
$\mathbf{7}$ & 1 & 0 & 0 & 1 & 0.1 & 0.5 \\
$\mathbf{8}$ & 1 & 0 & 0 & 1 & 0.5 & 0.1 \\
$\mathbf{9}$ & 1 & 0 & 0 & 1.05 & 0.1 & 0.5 \\
$\mathbf{1 0}$ & 1 & 0 & 0 & 1.18 & 0.1 & 0.5 \\
$\mathbf{1 1}$ & 1 & 10 & 10 & 1 & 0.1 & 0.5 \\
$\mathbf{1 2}$ & 1 & 10 & 2 & 1 & 0.1 & 0.5 \\
$\mathbf{1 3}$ & 1 & 10 & 20 & 1 & 0.1 & 0.5 \\
\hline
\end{tabular}

Table 1. Values of model parameters used in this study.

In Figures 4-13, the intersection points of the bisection line (denoted by a thin solid line) with the two curves are the fixed points.

\section{Discussion}

\subsection{Effect due to seismic coupling}

The lower-bound value of $\mathrm{s}$ for yielding strong coupling between the two sliders can be seen from Figures 4 and 5 , which are the simulation results for $s=5$ and $s=1$, respectively, when the values of other model parameters are equal. In each plot of Figure 4, the solid line is close to the dotted line, thus indicating strong coupling between the two sliders. Numerical tests also show the same phenomenon when $s>5$. Hence, the two sliders move almost simultaneously when $s \geq 5$, for which the system is self-organized. Figure 5 shows the results when $s=1$. In each plot the solid line is different from the dotted line, thus showing weak coupling between the two sliders. This phenomenon also exists when $s<5$. The peak values of $A / A_{\max }$ and $V / V_{\max }$ of slider 1 come earlier than those of slider 2 , while the peak value of $U / U_{\max }$ of the former comes later than that of the latter. The amplitudes of $A / A_{\max } V / V_{\max }$, and $U / U_{\max }$ on slider 2 are all larger than those on slider 1 . Results show the directivity of motions of sliders. Figures 4 and 5 suggest that strong coupling (with large s) between two fault segments is more capable of generating a larger-sized event than week coupling (with small $s$ ). Hence, for an earthquake fault consisting of a few segments it is easier to generate a larger-sized event from larger $s$ than from smaller $s$. Of course, the value of $s$ of generating a larger-sized event increases with the number of sliders [Wang, 1995]. From numerical simulations, Wang [1995] obtained a power-law correlation between the $b$-value of the Gutenberg-Richter frequency-magnitude law and $s: b \sim s^{-2 / 3}$ for the cumulative fre- quency and $b \sim s^{-1 / 2}$ for the discrete frequency. Larger $b$ for smaller $s$ is related to a bigger number of smaller events and smaller $b$ for larger $s$ to a bigger number of larger events. The present result is consistent with his. Figures $5 \mathrm{a}$ and $5 \mathrm{~b}$ reveal that the predominant periods of the two sliders are almost the same, because their values of model parameters are equal. In Figures $4 \mathrm{~d}$ and $5 \mathrm{~d}$, the absolute values of slope at the fixed points are likely both smaller than 1 and thus they can be an attractor.

\subsection{Effect due to Uc}

The effect due to $U_{c}$ can be seen from Figures 6-10. We examine the upper-bound values of $U_{c 1}$ and $U_{c 2}$ for allowing weak coupling between two sliders. In order to see the effect, $s=1$ is taken into account. Figure 6 shows the simulation results for $U_{c 1}=0.5$ and $U_{c 2}=0.5$ when the values of other model parameters are the same as those in Figure 5. In each plot the solid line is almost coincided with the dotted line, thus exhibiting strong coupling between the two sliders. Numerical tests also show the same phenomenon when $U_{c 1}>0.5$ and $U_{c 2}>0.5$. Hence, the two sliders move almost simultaneously when $U_{c 1} \geq 0.5$ and $U_{c 2} \geq 0.5$ at the two sliders. In Figure 6d, the fixed point for slider 1 is just the original point and thus it cannot be an attractor; and that for slider 2 is smaller than 1 and thus it can be an attractor.

Figure 7 shows the simulation results for $U_{c 1}=0.1$ and $U_{c 2}=0.5$ when the values of other model parameters are the same as those in Figure 6. In each plot the solid line is different from the dotted line. The peak values of $A / A_{\max }$ and $V / V_{\max }$ of slider 1 come earlier than those of slider 2, while the peak value of $U / U_{\max }$ of the former comes later than that of the latter. The peak values of $A / A_{\max }, V / V_{\max }$, and $U / U_{\max }$ of slider 1 are all lower than those of slider 2. The values of $U_{c 1}=0.1$ and $U_{c 2}=0.5$ are equivalent to $\gamma_{1}=10$ and $\gamma_{1}=2$, respectively. Hence, there is a larger force drop (or stress drop) on slider 1 than on slider 2, thus causing a large acceleration on slider 1 to push it to move. Then, the motion of slider 1 enforces slider 2 to move. Like Figure 5 , the directivity effect exists in the present case. In Figure $7 \mathrm{~d}$, the absolute values of slope at the fixed points are likely smaller than 1 for slider 1 and larger than 1 for slider 2 . Thus, the fixed point for the former can be an attractor, yet not for the latter.

Figure 8 shows the simulation results for $U_{c 1}=0.5$ and $U_{c 2}=0.1$ when the values of other model parameters are the same as those in Figures 6 and 7. Obviously, in each plot the solid line is different from the 

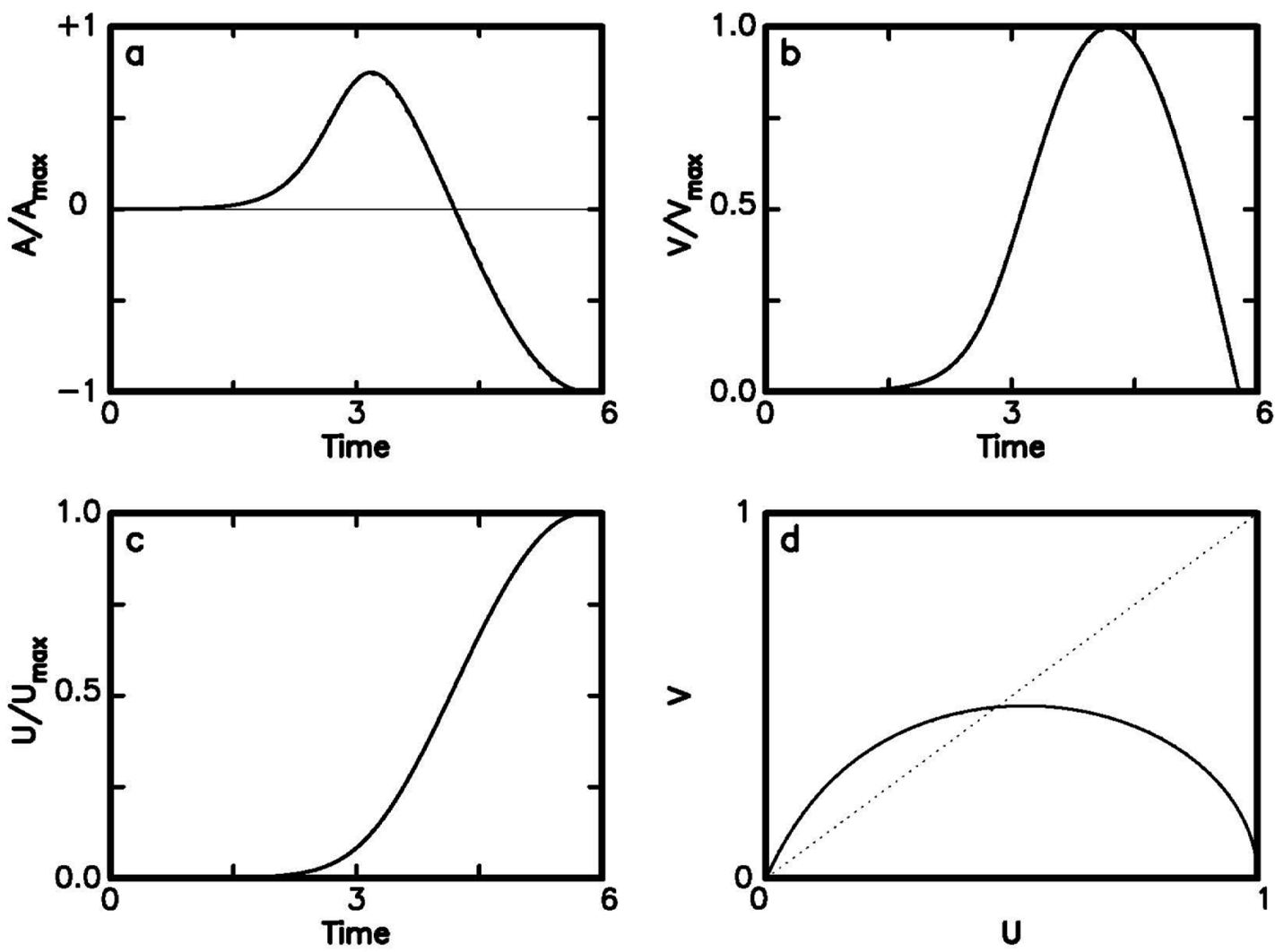

Figure 4. The time sequences of normalized acceleration $\left(A / A_{\max }\right)$, normalized velocity $\left(V / V_{\max }\right)$, and normalized displacement $(U /$ $U_{\max }$ and the phase portraits of $V$ versus $U$ of the two sliders (solid line for slider 1 and dashed line for slider 2 ) for $s=5, \eta_{1}=0, \eta_{2}=0$, $\varphi=1, \mathrm{U}_{\mathrm{c} 1}=0.1$, and $\mathrm{U}_{\mathrm{c} 2}=0.1$.

dotted line. Figure 8 is totally opposite to Figure 7 . The peak values of $A / A_{\max }$ and $V / V_{\max }$ of slider 1 come later than those of slider 2 , while the peak value of $U / U_{\max }$ of the former comes earlier than that of the latter. The peak values of $A / A_{\max }, V / V_{\max }$, and $U / U_{\max }$ of slider 1 are all higher than those of slider 2 . The values of $U_{c 1}=0.5$ and $U_{c 2}=0.1$ are equivalent to $\gamma_{1}=2$ and $\gamma_{2}=2$, respectively. Hence, there is a larger force drop (or stress drop) on slider 2 than on slider 1. Slider 1 moves first and then pushes slider 2 to move. A larger force drop (or stress drop) on slider 2 than on slider 1 makes a faster and bigger increase in acceleration on the former than on the latter. This makes the directivity effect do not exist in the present case. In Figure $8 \mathrm{~d}$, the absolute values of slope at the fixed points are likely larger than 1 for slider 1 and smaller than 1 for slider 2. Thus, the fixed point for the latter can be an attractor, yet not for the former.

\subsection{Effect due to static frictional force}

The effect of difference in $F_{o 1}$ and $F_{o 2}$ on the motions of two sliders is numerically made based on various values of $\varphi=F_{02} / F_{o 1}$ when the values of other model parameters are the same as those in Figure 7.
It is noted that since slider 1 is considered to be the first one to move, $\varphi$ must be equal to or larger than 1 . Results show that slider 2 cannot move when $\varphi \geq 1$.9. The value of $\varphi=1.9$ is the critical one for the present case. Of course, the critical value depends upon the values of other model parameters. For example, it somewhat increases with s. When $1.9>\varphi \geq 1.18$, the velocity of slider 1 increases, decreases, and reaches the minimum value when the velocity of slider 2 reaches its peak value. Then, slider 1 is pulled by slider 2 to move again. The separation of two events of slider 1 increases with $\varphi$. When $\varphi<1.18$, the velocity of both sliders increases, decreases, and becomes zero, and slider 1 cannot move again.

Figures 9 and 10 show the simulation results for $\varphi=1.05$ and $\varphi=1.18$, respectively, when the values of other model parameters are the same as those in Figure 7. Obviously, in each plot the solid line is different from the dotted line. Like Figure 7, the peak values of $A / A_{\max }$ and $V / V_{\max }$ of slider 1 come earlier than those at slider 2, while the peak value of $U / U_{\max }$ of the former comes later than that of the latter. The peak values of $A / A_{\max }, V / V_{\max }$, and $U / U_{\max }$ of slider 1 are all lower than those of slider 2. The reason to cause the results is the same as that for Figure 7. The difference 

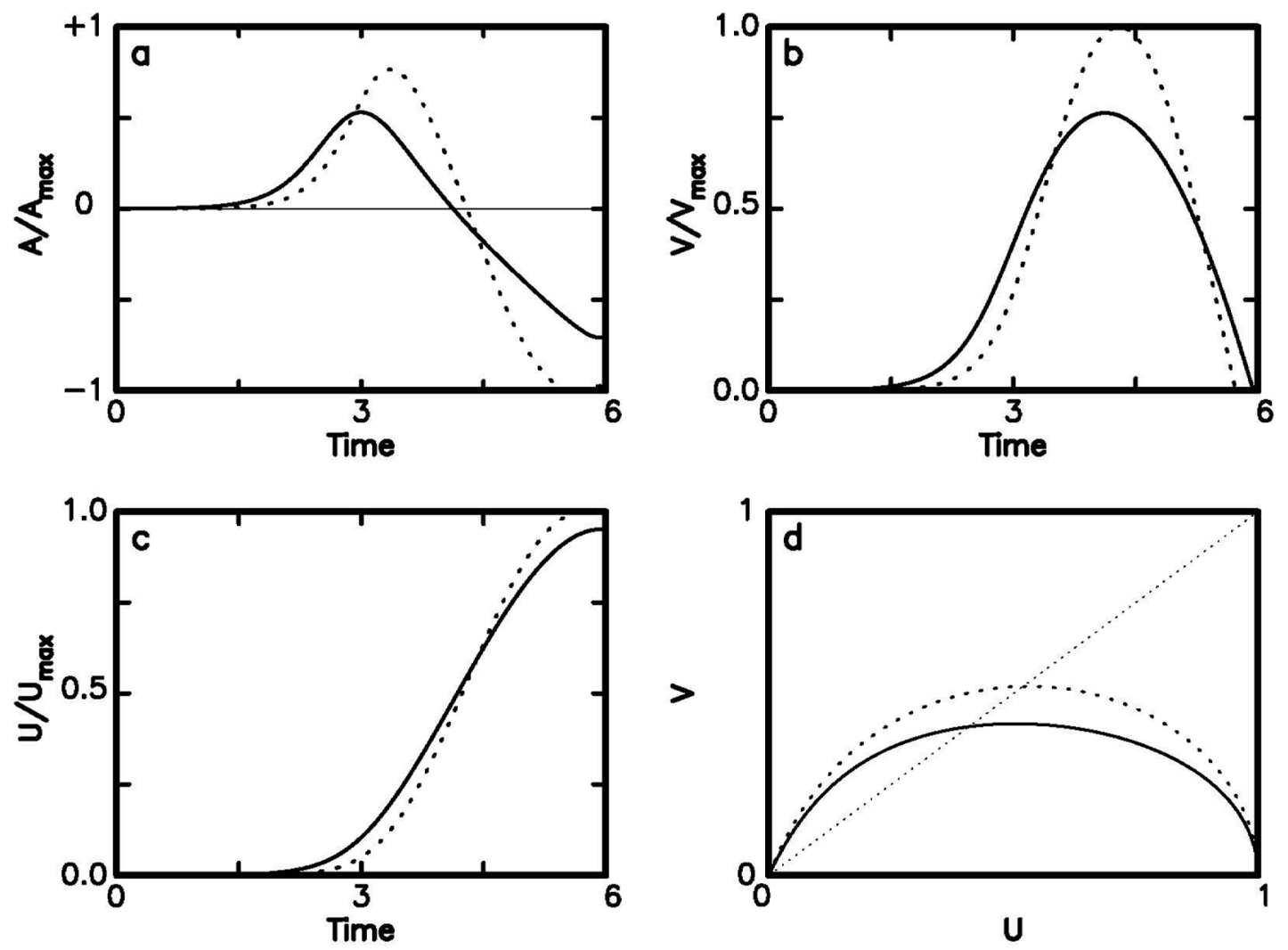

Figure 5. The time sequences of normalized acceleration $\left(A / A_{\max }\right)$, normalized velocity $\left(V / V_{\max }\right)$, and normalized displacement $\left(U / U_{\max }\right)$ and the phase portraits of $V$ versus $U$ of the two sliders (solid line for slider 1 and dashed line for slider 2 ) for $\mathrm{s}=5, \eta_{1}=0, \eta_{2}=0, \varphi=1, U_{\mathrm{cl}}=0.1$, and $U_{\mathrm{c} 2}=0.1$.
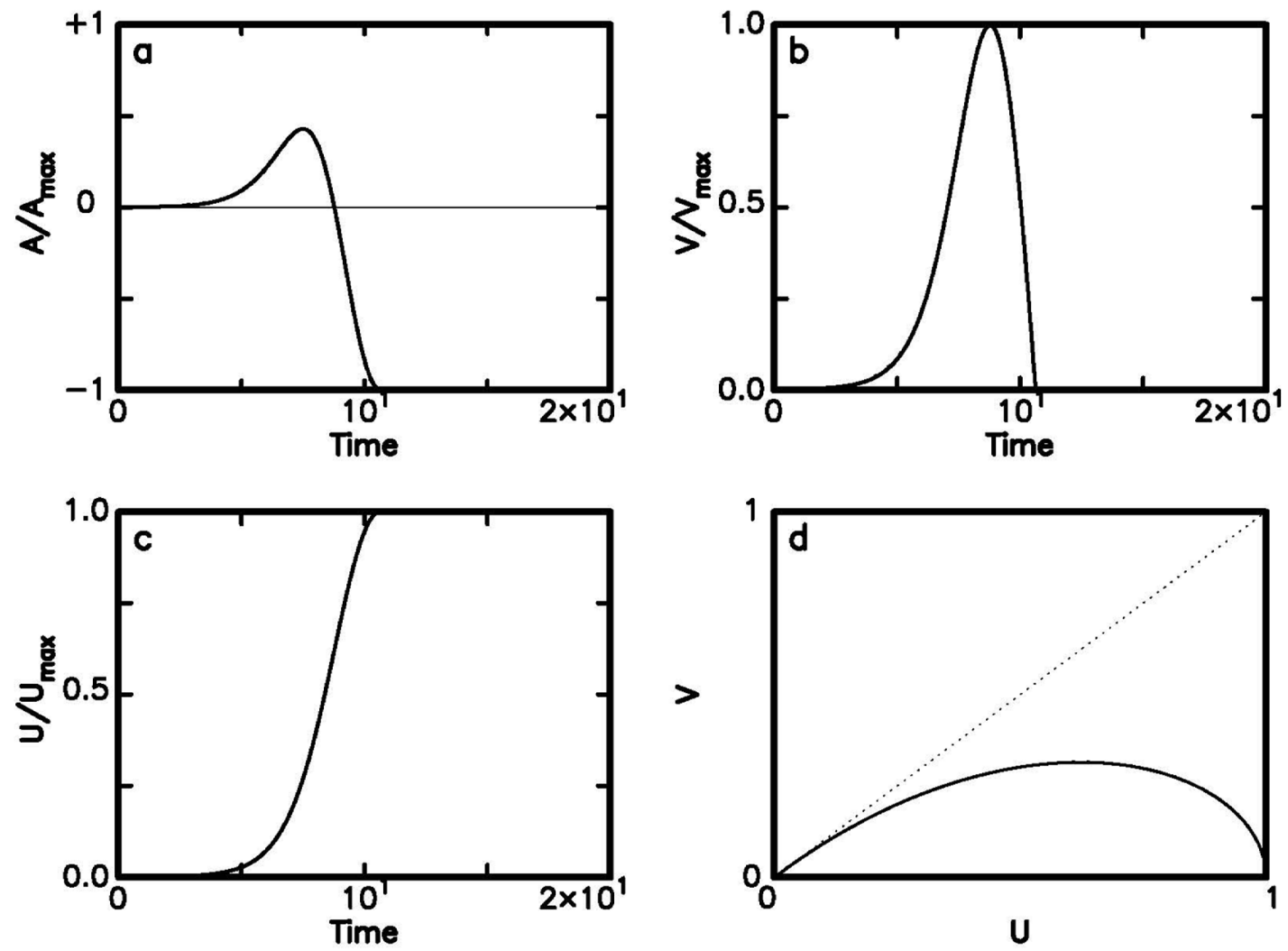

Figure 6. The time sequences of normalized acceleration $\left(A / A_{\max }\right)$, normalized velocity $\left(V / V_{\max }\right.$, and normalized displacement $\left(U / U_{\max }\right)$ and the phase portraits of $V$ versus $U$ of the two sliders (solid line for slider 1 and dashed line for slider 2) for $s=1, \eta_{1}=0, \eta_{2}=0, \varphi=1, U_{c 1}=0.5$, and $U_{c 2}=0.5$. 

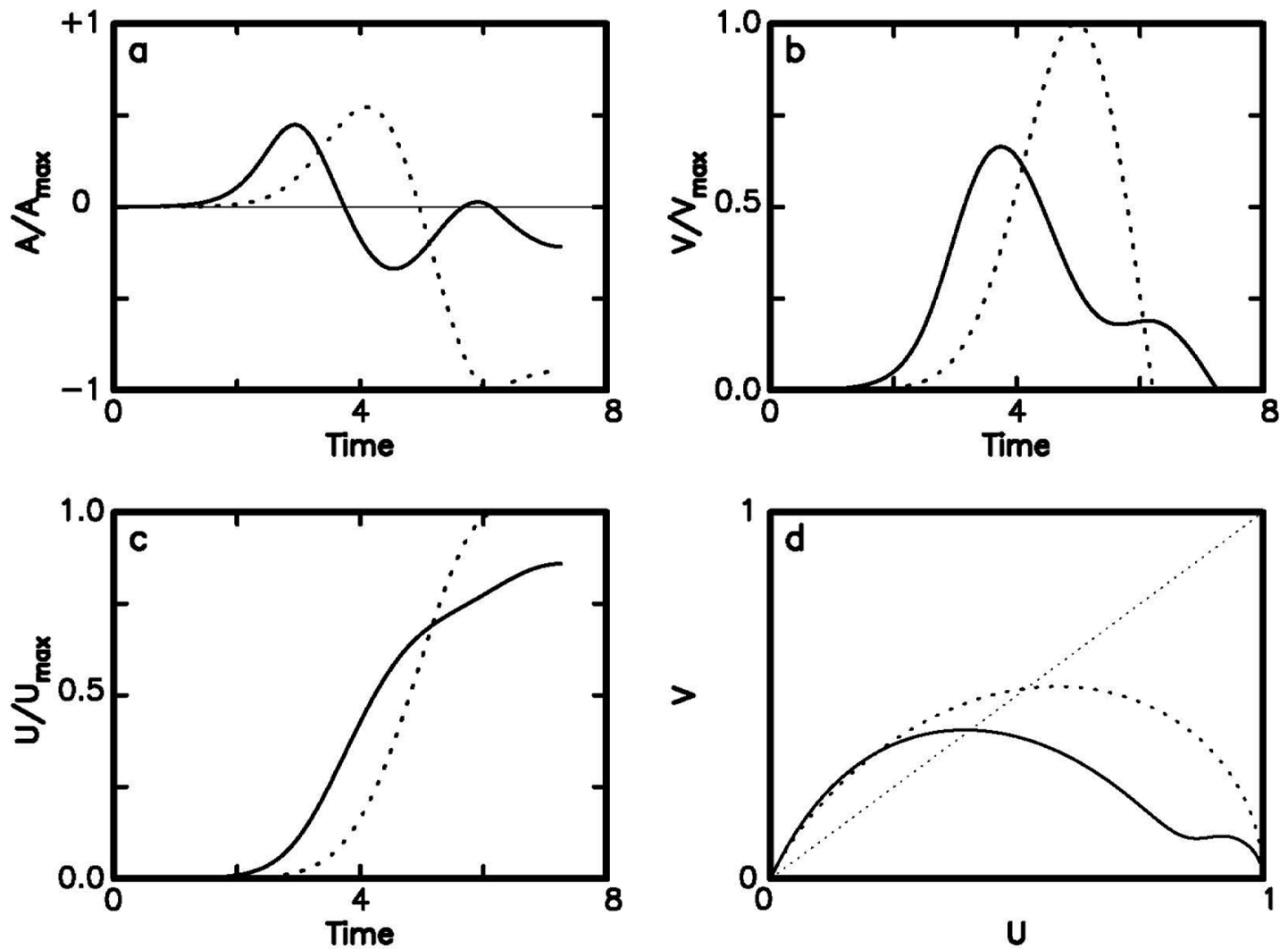

Figure 7. The time sequences of normalized acceleration $\left(A / A_{\max }\right)$, normalized velocity $\left(V / V_{\max }\right)$, and normalized displacement $\left(U / U_{\max }\right)$ and the phase portraits of $V$ versus $U$ of the two sliders (solid line for slider 1 and dashed line for slider 2 ) for $s=1, \eta_{1}=0, \eta_{2}=0, \varphi=1, U_{c 1}=0.1$, and $U_{c 2}=0.5$.
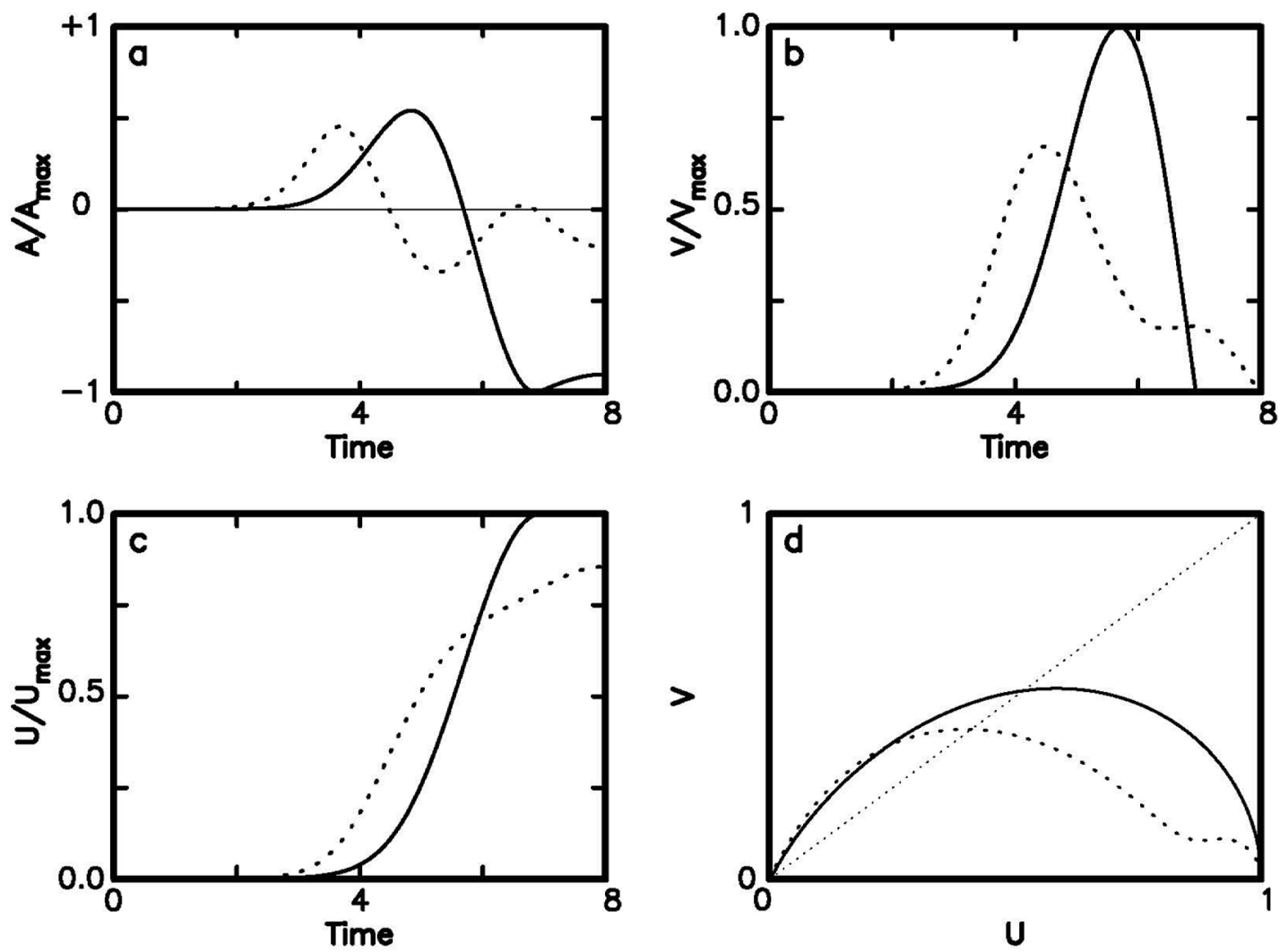

Figure 8. The time sequences of normalized acceleration $\left(A / A_{\max }\right.$, normalized velocity $\left(V / V_{\max }\right)$, and normalized displacement $\left(U / U_{\max }\right)$ and the phase portraits of $V$ versus $U$ of the two sliders (solid line for slider 1 and dashed line for slider 2 ) for $s=1, \eta_{1}=0, \eta_{2}=0, \varphi=1, U_{c 1}=0.5$, and $U_{c 2}=0.1$. 

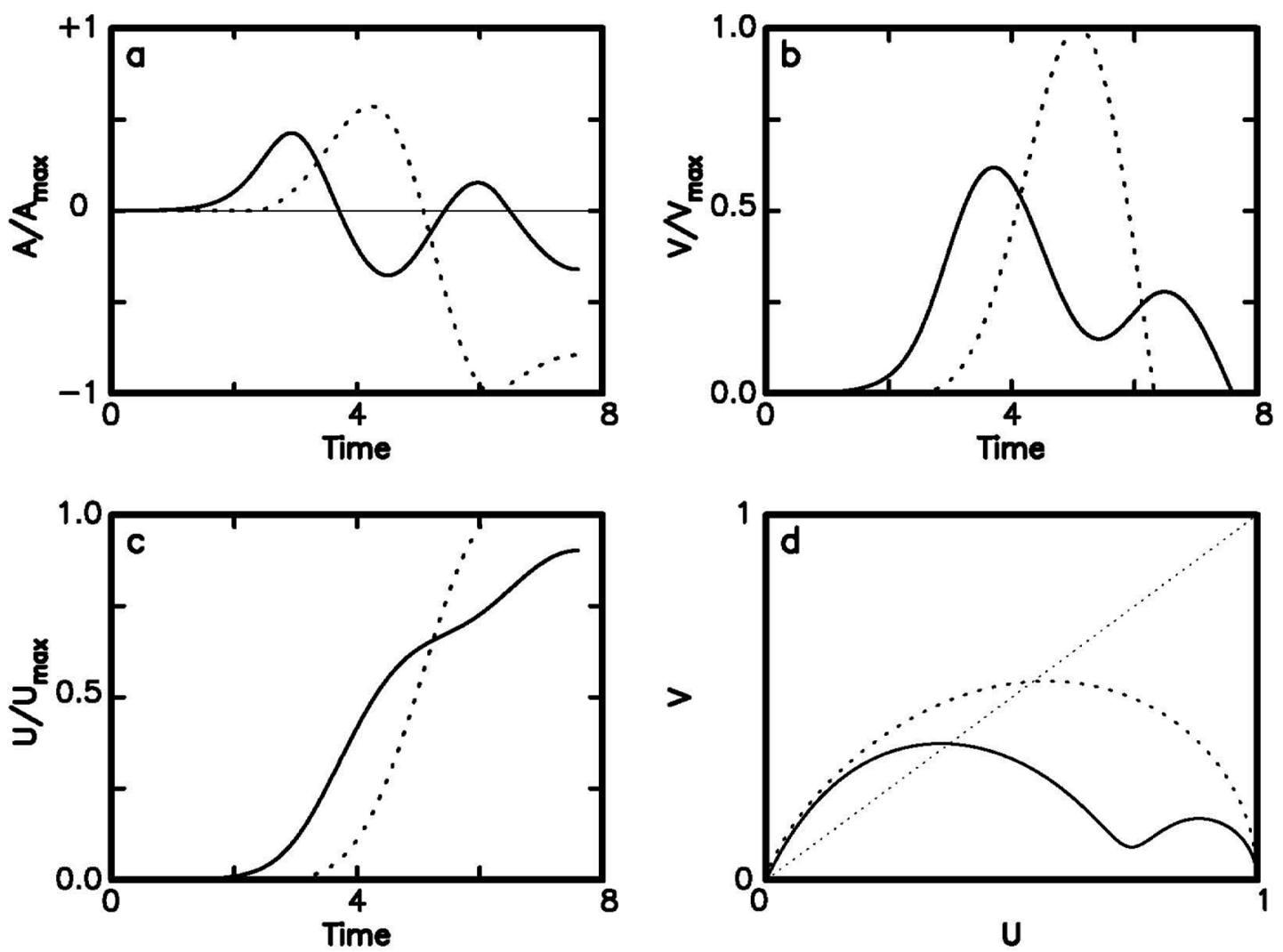

Figure 9. The time sequences of normalized acceleration $\left(A / A_{\max }\right.$, normalized velocity $\left(V / V_{\max }\right)$, and normalized displacement $\left(U / U_{\max }\right)$ and the phase portraits of $V$ versus $U$ of the two sliders (solid line for slider 1 and dashed line for slider 2 ) for $s=1, \eta_{1}=0, \eta_{2}=0, \varphi=1, U_{c 1}=0.1$, and $U_{c 2}=0.5$.
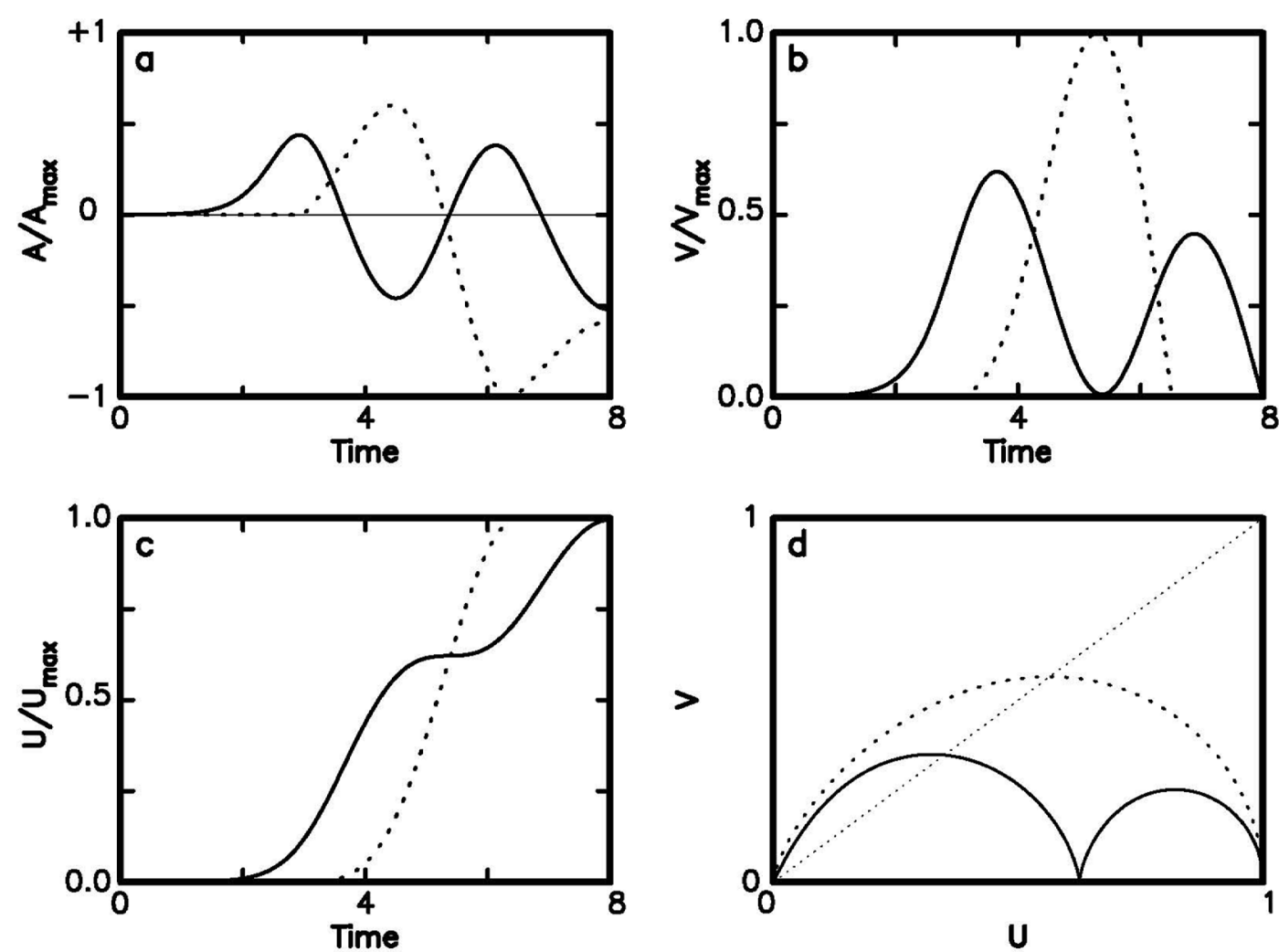

Figure 10. The time sequences of normalized acceleration $\left(A / A_{\max }\right)$, normalized velocity $\left(V / V_{\max }\right)$, and normalized displacement $\left(U / U_{\max }\right)$ and the phase portrait of $V$ versus $U$ of the two sliders (solid line for slider 1 and dashed line for slider 2)r for $s=1, \eta_{1}=0, \eta_{2}=0, \varphi=1.18$, $U_{c 1}=0.1$, and $U_{c 2}=0.5$. 

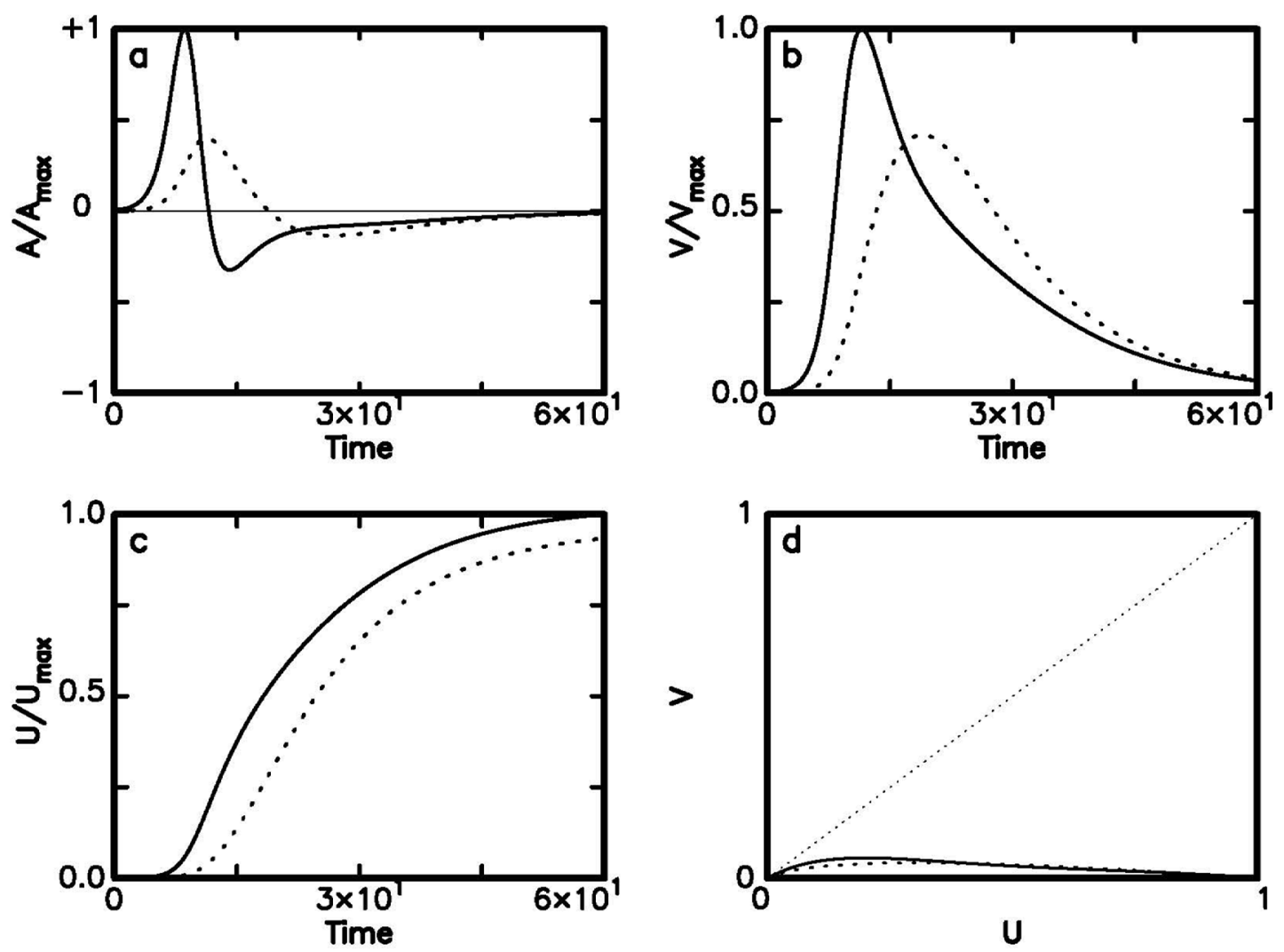

Figure 11. The time sequences of normalized acceleration $\left(A / A_{\max }\right)$, normalized velocity $\left(V / V_{\max }\right)$, and normalized displacement $(U /$ $U_{\max }$ ) and the phase portraits of $V$ versus $U$ of the two sliders (solid line for slider 1 and dashed line for slider 2) for $s=1, \eta_{1}=10, \eta_{2}=10, \varphi=1$, $U_{c 1}=0.1$, and $U_{c 2}=0.5$.
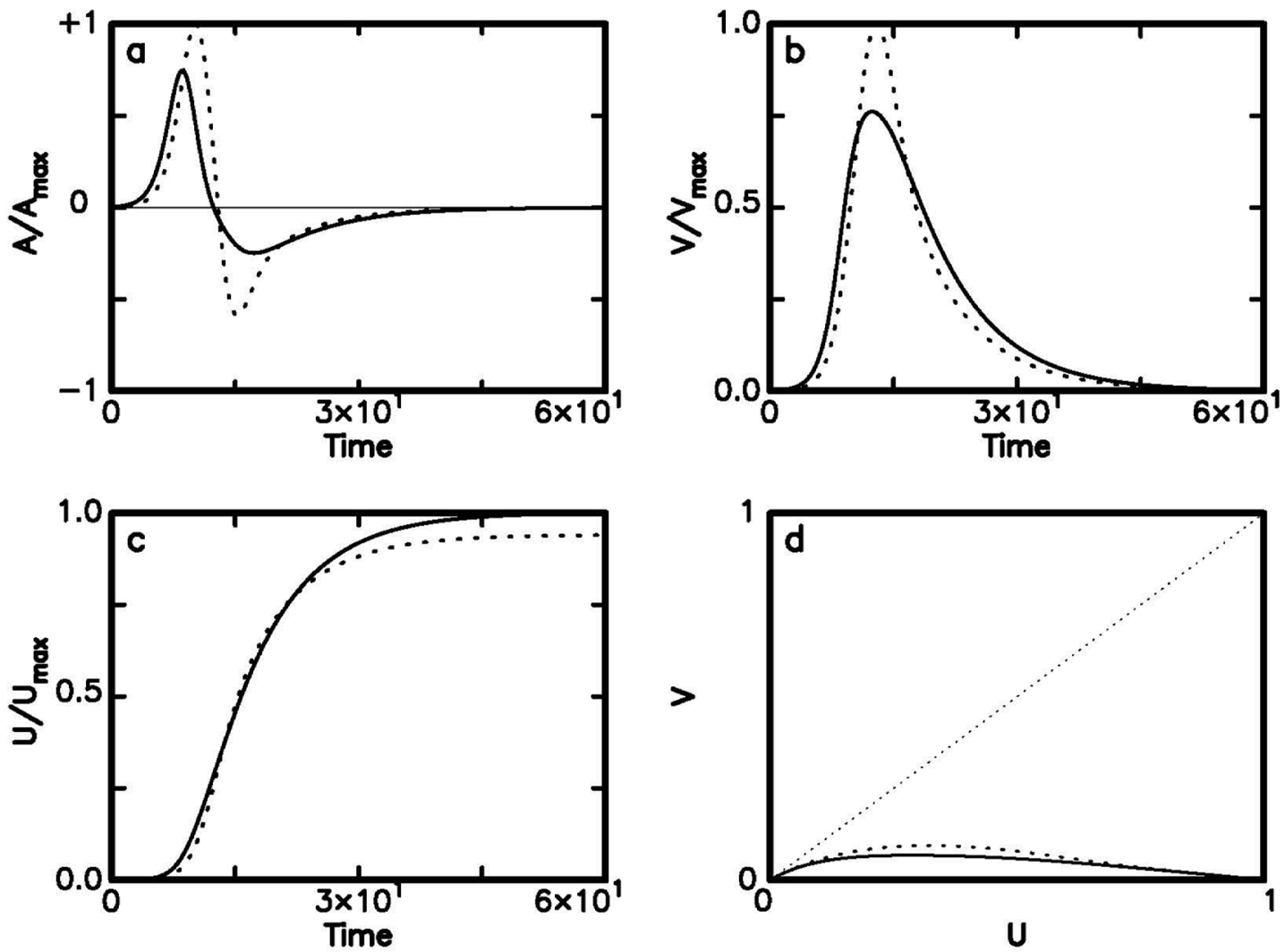

Figure 12. The time sequences of normalized acceleration $\left(A / A_{\max }\right)$, normalized velocity $\left(V / V_{\max }\right)$, and normalized displacement $(V /$ $V_{\max }$ ) and the phase portraits of $v$ versus $U$ of the two sliders (solid line for slider 1 and dashed line for slider 2) for $s=1, \eta_{1}=10, \eta_{2}=2, \varphi=1$, $U_{c 1}=0.1$, and $U_{c 2}=0.5$. 

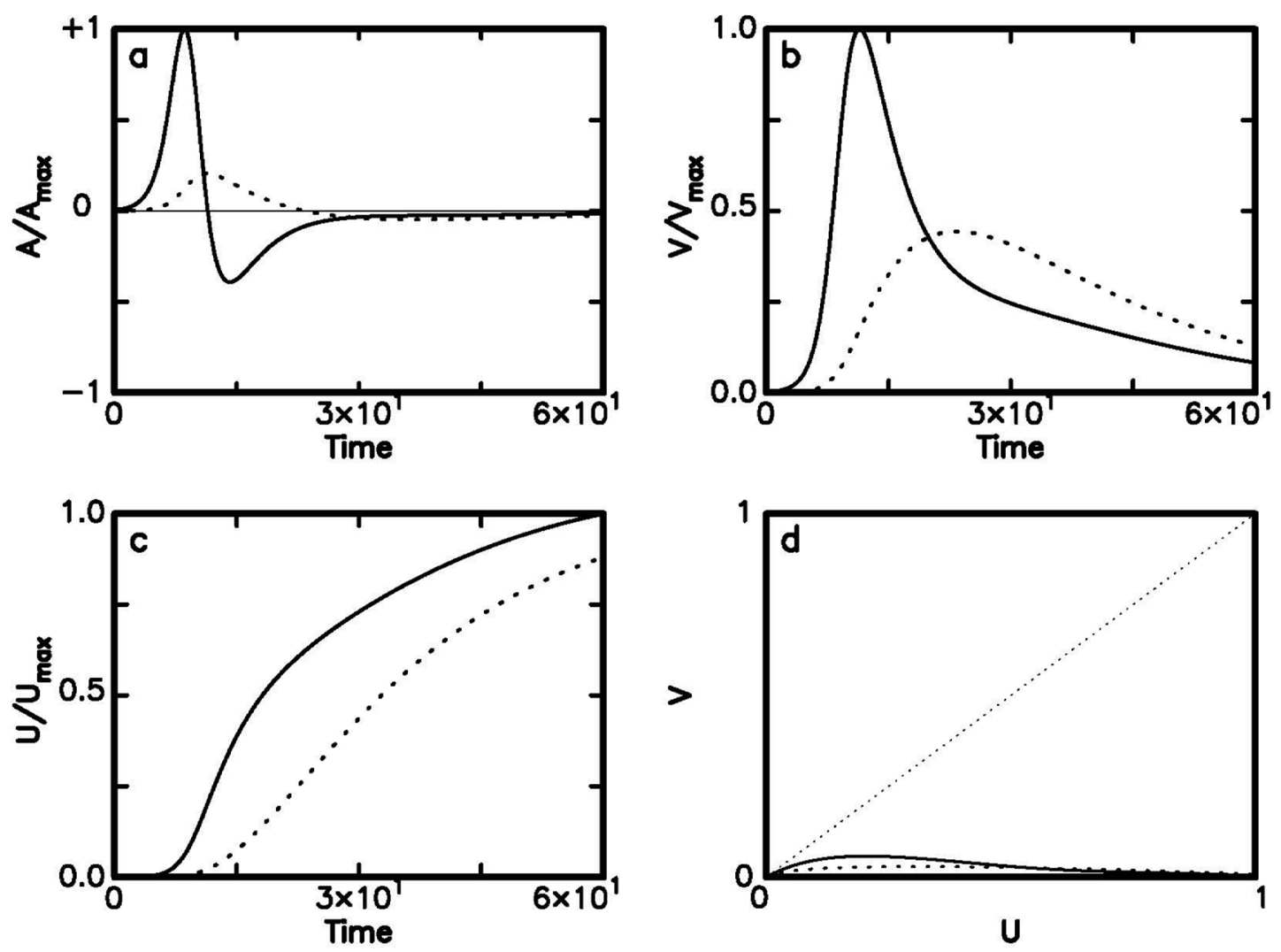

Figure 13. The time sequences of normalized acceleration $\left(A / A_{\max }\right)$, normalized velocity $\left(V / V_{\max }\right)$, and normalized displacement $(U /$ $U_{\max }$ ) and the phase portraits of $V$ versus $U$ of the two sliders (solid line for slider 1 and dashed line for slider 2 ) for $s=1, \eta_{1}=10, \eta_{2}=20, \varphi=1$, $U_{c 1}=0.1$, and $U_{c 2}=0.5$.

between Figure 7 and Figure 9 as well as Figure 10 is that the increases in $A / A_{\max }, V / V_{\max }$, and $U / U_{\max }$ come slightly later for Figures 9 and 10 than for Figure 7, because it takes a longer time to increase the force on slider 2 from driving force due to $\varphi>1$ or $\mathrm{F}_{\mathrm{o} 2}>\mathrm{F}_{\mathrm{o} 1}$. Obviously, there are two bumps in the temporal variation in velocity of slider 1. In Figure 9d, the absolute values of slope at the fixed points are likely smaller than 1 for slider 1 and larger than 1 for slider 2. Thus, the fixed point for the former can be an attractor, yet not for the latter. In Figure 10d, the absolute values of slope at the fixed points are likely smaller than 1 and thus they can be an attractor. Clearly, $\varphi$ can influence the generation an attractor in slider 2 .

Figures 4-10 show that nonlinear slip-dependent friction can result in an attractor for chaotic slip in the model. This is similar to the chaotic motions in a two-degree-of-freedom system studied by numerous researches [e.g., Huang and Turcotte, 1990a,b, 1992; de Sousa Viera, 1999; and Abe and Kato, 2013].

\subsection{Effect due to viscosity}

Figures 11-13 show the simulation results for $\eta_{1}=10$ and $\eta_{2}=10, \eta_{1}=10$ and $\eta_{2}=2$, and $\eta_{1}=10$ and $\eta_{2}=20$, respectively, when the values of other model parameters are the same as those in Figure 7. Obviously, in each plot the solid line is different from the dotted line. In Figure 11, the peak values of $A / A_{\max } V /$ $V_{\max }$, and $U / U_{\max }$ of slider 1 come earlier than those of slider 2. The peak values of $A / A_{\max }, V / V_{\max }$, and $U / U_{\max }$ of slider 1 are all lower than those of slider 2. The peak values of $A / A_{\max } V / V_{\max }$, and $U / U_{\max }$ are all smaller at slider 1 than at slider 2 in Figure 7, yet opposite in Figure 2. This indicates that viscosity can affect the amplitudes of the three quantities. In Figure 12, the peak value of $A / A_{\max }$ comes slightly earlier at slider 1 than at slider 2; while the peak value of $V / V_{\max }$ comes slightly later at slider 1 than at slider 2. The peak values of $A / A_{\max }$ and $V / V_{\max }$ of slider 1 are lower than those of slider 2 . The value of $U / U_{\max }$ of slider 1 is first similar to and then larger than that of slider 2. In Figure 13, the peak values of $A / A_{\max }$ and $V / V_{\max }$ of slider 1 come earlier than those at slider 2 . The peak values of $A / A_{\max }$ and $V / V_{\max }$ of slider 1 are larger than those of slider 2 . The value of $U / U_{\max }$ of slider 1 is larger than that of slider 2 .

Unlike Figures 4-10, the duration times in Figures 11-13 become longer due to the viscous effect. Since weak coupling (with $s=1$ ) between the two sliders, their motions can be considered to be somewhat in- 
dependent. Wang [2016b] studied the viscous effect on the predominant period of a one-degree-of-freedom spring-slider model. Here, a study of the viscous effect on the predominant periods of the two-degree-of-freedom spring-slider model is conducted. From Equation (1), the natural period of each slider is $T_{0}=2 \pi(\mathrm{m} / L)^{1 / 2}$ in the absence of friction and viscosity. When the two sliders are linked together, the natural period of each slider must be slightly different from $T_{0}$. When viscosity is present, the natural period is $T_{1}=T_{o 1} /\left(1-C_{1}^{2} / 4 m L\right)$ for slider 1 and $T_{2}=T_{o 2} /\left(1-C_{2}{ }^{2} / 4 m L\right)$ for slider 2 . Obviously, viscosity produces damping and increases the predominant period of oscillations of the slider. The system is under-damping, critical damping, and over-damping when $C_{i}^{2} / 4 m L<1, C_{i}^{2} / 4 m L=1$, and $C_{i}^{2} / 4 m L>1$, respectively. Since $T_{01}=T_{o 2}$ in this study, the ratio of $T_{2}$ to $T_{1}$ is:

$$
\mathrm{T}_{2} / \mathrm{T}_{1}=\left[\left(\alpha-C_{1}^{2}\right) /\left(\alpha-C_{2}^{2}\right)\right] 1 / 2
$$

where $\alpha=4 m L$. In Figure 11, $T_{1} \approx T_{2}$ because of $C_{1}=C_{2}$ from $\eta_{1}=\eta_{2}$. In Figure 12, $T_{1}>T_{2}$ because of $C_{1}>C_{2}$ from $\eta_{1}>\eta_{2}$. In Figure 13, $T_{1}<T_{2}$ because of $C_{1}<C_{2}$ from $\eta_{1}<\eta_{1}$. In addition, the values of $A / A_{\max }, V /$ $V_{\max }$, and $U / U_{\max }$ with viscosity are smaller than those without viscosity, because viscosity can decrease the amplitude of vibration. The viscous effect on slip of the model is consistent with that mentioned by Wang [2007] who applied the model to study the difference in ground motions between the northern and southern segments of the Chelungpu fault generated by the 1999 Chi-Chi, Taiwan, $M_{s} 7.6$ earthquake.

In Figures $11 \mathrm{~d}, 12 \mathrm{~d}$, and $13 \mathrm{~d}$, the fixed points are just the original point and thus they cannot be an attractor. Hence, the presence of viscosity between the slider and moving plate can depress the generation of attractor with $\left|f^{\prime}\left(x_{f}\right)\right|<1$. Consequently, viscosity plays a role on preventing chaotic behavior of earthquake ruptures.

In addition, a comparison between the cases in the absence of viscosity (Figures 4-10) and those in the presence of viscosity (Figures 11-13) shows that the presence of viscosity results in smaller velocities of sliders than the absence of viscosity. This suggests that viscosity can cause slow earthquakes or creep of faults.

\section{Conclusions}

Simulation results show that slip of the slider are affected by seismic coupling, friction (including the characteristic displacement of the friction law and the static frictional force), and viscosity. For seismic coupling, the coupling between the sliders is weak when $s<5$ and strong when $s \geq 5$. For characteristic displacement of the friction law the characteristic displacement of the friction law, there are two concluding points: (1) The coupling between the two sliders is weak when $U_{\mathrm{c} 1}$ and $U_{\mathrm{c} 2}$ are both equal to or smaller than 0.5 ; and (2) The motions of the two sliders yielded by large $U_{\mathrm{c} 1}$ and small $U_{\mathrm{c} 2}$ are opposite to those by small $U_{\mathrm{c} 1}$ and large $U_{\mathrm{c} 2}$. For the difference on static frictional forces, a higher static friction force at slider 2 causes a longer delay of its motion; and slider 2 cannot move when its static friction force is higher than a critical value which will depend on other model parameters. For the viscosity between a slider and the moving plate, there are four concluding points: (1) Viscosity results in increases in duration times and predominant periods of motions of sliders; (2) Higher viscosity decreases the amplitude of motion; (3) Viscosity causes a decrease in velocities of sliders; and (4) Viscosity depresses the generation of attractors, which can lead to chaotic behavior of the system.

Acknowledgements. The author thanks an anonymous reviewer and Dr. Andrea Bizzarri (Associated Editor of the journal) for useful comments and suggestions to improve the article. The study was financially supported by Academia Sinica, the Ministry of Science and Technology (Grant No.: MOST-1042116-M-001-007), and the Central Weather Bureau (Grant Nos.: MOTC-CWB-105-E-02 and MOTC-CWB- 106-E-02).

\section{References}

Abe and Kato (2013). Complex earthquake cycle simulations using a two-degree- of-freedom spring-block model with a rate- and state-friction law. Pure Appl. Geophys., 170, 745-765.

Ampuero, J.P. and A.M. Rubin (2008). Earthquake nucleation on rate and state faults $\sim$ Aging and slip laws. J. Geophys. Res., 113, B01302, doi:10.1029/ 2007JB005082.

Bhattacharya, A. and A.M. Rubin (2014). Frictional response to velocity steps and 1-D fault nucleation under a state evolution law with stressing-rate dependence. J. Geophys. Res. Solid Earth, 119, 22722304, doi:10.1002/2013JB010671.

Bizzarri, A. (2009). Can flash heating of asperity contacts prevent melting?. Geophys. Res. Lett., 36, L11304, doi:10.1029/2009GL037335.

Bizzarri, A. (2010). An efficient mechanism to avert frictional melts during seismic ruptures. Earth Planet. Sci. Lett., 296, 144-152, doi:10.1016/j. epsl.2010.05.012.

Bizzarri, A. (2011a). Dynamic seismic ruptures on melting fault zones. J. Geophys. Res., 116, B02310, doi:10.1029/2010JB007724. 
Bizzarri, A. (2011b). Temperature variations of constitutive parameters can significantly affect the fault dynamics. Earth Planet. Sci. Lett., 306, 272-278, doi: 10.1016/j.epsl.2011.04.009.

Bizzarri, A. (2011c). On the deterministic description of earthquakes. Rev. Geophys., 49, RG3002, doi:10.1029/2011RG000356.

Bizzarri, A. and M. Cocco (2006a). A thermal pressurization model for the spontaneous dynamic rupture propagation on a three-dimensional fault: 1 . Methodological approach. J. Geophys. Res., 111, B05303, doi:10.1029/ 2005JB003862.

Bizzarri, A., and M. Cocco (2006b). A thermal pressurization model for the spontaneous dynamic rupture propagation on a three-dimensional fault: 2 . Traction evolution and dynamic parameters. J. Geophys. Res., 111, B05304, doi:10.1029/2005JB003864.

Bizzarri, A. and M. Cocco (2006c). Correction to "A thermal pressurization model for the spontaneous dynamic rupture propagation on a three-dimensional fault: 1. Methodological approach", J. Geophys. Res., 111, B11302, doi:10.1029/ 2006JB004759.

Burridge, R. and L. Knopoff (1967). Model and theoretical seismicity. Bull. Seism. Soc. Am., 57, 341-371.

Byerlee, J.D. (1968). Brittle-ductile transition in rocks. J. Geophys. Res., 73, 4711-4750.

Byerlee, J. (1978). Friction of rocks. Pure. Appl. Geophys., 116, 615-626.

Cao, T. and K. Aki (1984/85). Seismicity simulation with a mass-spring model and a displacement hardening-softening friction law. Pure. Appl. Geophys., 122, 10-24.

Carlson, J.M. and J.S. Langer (1989). Mechanical model of an earthquake fault. Phys. Rev. A, 40, 6470-6484.

Carlson, J.M., J.S. Langer, B.E. Shaw, and C. Tang (1991). Intrinsic properties of a Burridge-Knopoff model of an earthquake fault. Phys. Rev. A, 44, 884897.

Cochard, A. and R. Madariaga (1994). Dynamic faulting under rate-dependent friction. Pure Appl. Geophys., 142, 419-445, doi:10.1007/BF00876049.

Cohen, S. (1979). Numerical and laboratory simulation of fault motion and earthquake occurrence. Rev. Geophys. Space Phys., 17(1), 61-72

Dieterich, J.H. (1972). Time-dependent friction in rocks. J. Geophys. Res., 77(20), 3690-3697.

Dieterich, J.H. (1979). Modeling of rock friction 1. Experimental results and constitutive equations. J. Geophys. Res., 84, 2161-2168.

Dragoni, M. and S. Santini (2010). Simulation of the long-term behaviour of a fault with two asperities.
Nonlin. Proc. Geophys., 17, 777-784.

Dragoni, M. and S. Santini (2011). Conditions for large earthquakes in a two-asperity fault model. Nonlin. Process. Geophys., 18, 709-717.

Dragoni, M. and S. Santini (2012). Long-term dynamics of a fault with two asperities of different strengths. Geophys. J. Int., 191, 1457-1467.

Dragoni, M. and S. Santini (2014). Source functions of a two-asperity fault model. Geophys. J. Int., 196, 1803-1812.

Fialko, Y.A. (2004). Temperature fields generated by the elastodynamic propagation of shear cracks in the Earth. J. Geophys. Res., 109, B01303, doi:10.1029/ 2003JB002496.

Galvanetto, U. (2002). Some remarks on the two-block symmetric Burridge-Knopoff model. Phys. Letts. A, 293, 251-259.

Hainzl, S., G. Zoller, and J. Kurths (1999). Similar power laws for foreshock and aftershock sequences in a spring-block model for earthquakes. J. Geophys. Res., 104(B4), 7243-7253.

He, C., S. Ma, and J. Huang (1998). Transition between stable sliding and stick-slip due to variation in slip rate under variable normal stress condition. Geophys. Res. Letts., 25(17), 3235-3238.

Huang, J. and D.L. Turcotte (1990a). Are earthquakes an example of deterministic chaos?. Geophys. Res. Lett., 17, 223-226.

Huang, J. and D.L. Turcotte (1990b). Evidence for chaotic fault interactions in the seismicity of the San Andreas Fault and Nankai Trough. Nature, 348, 234-236.

Huang, J. and D.L. Turcotte (1992). Chaotic seismic faulting with a mass-spring model and velocity-weakening friction. Pure Appl. Geophys., 138, 569-589.

Hudson, J.A. (1980). The excitation and propagation of elastic waves. Cambridge Monographs on Mechanics and Applied Mathematics, Cambridge Univ. Press, 224pp.

Ionescu, I.R. and M. Campillo (1999). Influence of the shape of the friction law and fault finiteness on the duration of initiation. J. Geophys. Res., 104(B2), 3013-3024.

Jaeger, J.C. and N.G.W. Cook (1977). Fundamentals of Rock Mechanics. John Wiley \& Sons, Inc., New York, 585pp.

Jeffreys, H. (1942). On the mechanics of faulting. Geol. Mag., 79, 291-295.

Kittel, C., W.D. Knight, and M.A. Ruderman (1968). Mechanics. Berkeley Physics Course Volume 1, 
McGraw-Hill Book Co., New York, N.Y., 480pp.

Knopoff, L. and X.X. Ni (2001). Numerical instability at the edge of a dynamic fracture. Geophys. J. Int., 147, F1-F6.

Knopoff, L., J.Q. Mouton, and R. Burridge (1973). The dynamics of a one-dimensional fault in the presence of friction. Geophys. J. R. astro. Soc., 35, 169184.

Knopoff, L., J.A. Landoni, and M.S. Abinante (1992). Dynamical model of an earthquake fault with localization. Phys. Rev. A, 46, 7445-7449.

Lu, X., A.J. Rosakis, and N. Lapusta (2010). Rupture modes in laboratory earthquakes: Effect of fault prestress and nucleation conditions. J. Geophys. Res., 115, B12302, doi:10.1029/2009JB006833.

Madariaga, R., and A. Cochard (1994). Seismic source dynamics, heterogeneity and friction. Ann. Geofis., 37(6), 1349-1375.

Marone, C. (1998). Laboratory-derived friction laws and their application to seismic faulting. Ann. Rev. Earth Planet. Sci., 26, 643-669.

Noda, H., E.M. Dunham, and J.R. Rice (2009). Earthquake ruptures with thermal weakening and the operation of major faults at low overall stress levels. J. Geophys. Res., 114, B07302, doi:10.1029/ 2008JB006143.

Nur, A. (1978). Nonuniform friction as a physical basis for earthquake mechanics. Pure Appl. Geophys., 116, 964-989.

Ohnaka, M. (2003). A constitutive scaling law and a unified comprehension for frictional slip failure, shear fracture of intact rocks, and earthquake rupture. J. Geophys. Res., 108, B2, 2080, doi:10.1029/ 2000JB000123.

Press, W.H., B.P. Flannery, S.A. Teukolsky and W.T. Vetterling (1986). Numerical Recipes. Cambridge Univ. Press, Cambridge, 818pp.

Rice, J.R. (1993). Spatio-temporal complexity of slip on a fault. J. Geophys. Res., 98(B6), 9885-9907.

Rice, J.R. (2006). Heating and weakening of faults during earthquake slip. J. Geophys. Res., 111, B05311, doi:10.1029/2005JB004006.

Rice, J.R., N. Lapusta, and K. Ranjith (2001). Rate and state dependent friction and the stability of sliding between elastically deformable solids. J. Mech. Phys. Solids, 49, 1865-1898.

Rubin, A.M. and J.P. Ampuero (2005). Earthquake nucleation on (aging) rate and state faults. J. Geophys. Res., 110, B11312, doi:10.1029/2005JB003686.

Ruina, A.L. (1983). Slip instability and state variable friction laws. J. Geophys. Res., 88, 10359-10370.
Ryabov, V.B. and K. Ito (2001). Intermittent phase transitions in a slider-block model as a mechanism for earthquakes. Pure Appl. Geophys., 158, 919-930.

Savage, J.C. and A.H. Lachenbruch (2003). Consequences of viscous drag beneath a transform fault. J. Geophys. Res., 108(B1), 2025, doi:10.1029/ 2001JB000711.

Shaw, B.E. (1994). Complexity in a spatially uniform continuum fault model. Geophys. Res. Lett., 21, 1983-1986.

Scholz, C.H. (1990). The Mechanics of Earthquakes and Faulting. Cambridge Univ. Press, Cambridge, 439pp.

Shimamoto, T. (1986). Strengthening of phyllosilicate and gypsum gouges with increasing temperature: effect of temperature or moisture elimination?. Int. J. Rock Mech. Min. Sci. \& Geomech. Abstr., 123, 439-443.

Sibson, R.H. (1973). Interaction between temperature and pore-fluid pressure during earthquake faulting and a mechanism for partial or total stress relief. Natural Phys. Sci., 243, 66-68.

Sibson, R.H. (1975). Generation of pseudotachylite by ancient seismic faulting. Geophys. J. Roy. Astr. Soc., 43, 775-794.

Sone, H. and T. Shimamoto (2009). Frictional resistance of faults during accelerating and decelerating earthquake slip. Nat. Geosci., 2, 705-708, doi:10.1038/ngeo637.

de Sousa Viera, M. (1999). Chaos and synchronized chaos in an earthquake model. Phys. Rev. Lett., 82, 201-204.

Spray, J.G. (1993). Viscosity determinations of some frictionally generated silicate melts: Implications for fault zone rheology at high strain rates. J. Geophys. Res., 98(B5), 8053-8068.

Spray, J.G. (1995). Pseudotachylyte controversy: Fact or friction?. Geology, 23(12), 1119-1122.

Spray, J.G. (2005). Evidence for melt lubrication during large earthquakes. Geophys. Res. Lett., 32, L07301, doi:10.1029/2004GL022293.

Stuart, W.D. (1981). Stiffness method for anticipating earthquakes. Bull. Seism. Soc. Am., 71, 363-370.

Thompson, J.M.T. and H.B. Stewart (1986). Nonlinear Dynamics and Chaos. John Wiley and Sons, New York, 376pp.

Turcotte, D.L. (1992). Fractals and Chaos in Geology and Geophysics. Cambridge University Press, $221 \mathrm{pp}$.

Turcotte, D.L. and G. Schubert (1982). GEODYNAMICS - Applications of Continuum Physics to Geo- 
logical Problems. Wiley, 450pp.

Urata, Y., K. Kuge, and Y. Kase (2008). Heterogeneous rupture on homogeneous faults: Three-dimensional spontaneous rupture simulations with thermal pressurization. Geophys. Res. Letts., 35, L21307, doi:10.1029/2008GL035577.

Wang, J.H. (1995). Effect of seismic coupling on the scaling of seismicity. Geophys. J. Int., 121, 475-488.

Wang, J.H. (1996). Velocity-weakening friction law as a factor in controlling the frequency-magnitude relation of earthquakes. Bull. Seism. Soc. Am., 86, 701-713.

Wang, J.H. (1997). Effect of frictional healing on the scaling of seismicity. Geophys. Res. Lett., 24, 25272530 .

Wang, J.H. (2000). Instability of a two-dimensional dynamical spring-slider model of an earthquake fault. Geophys. J. Int., 143, 389-394.

Wang, J.H. (2002). A dynamical study of comparing two one-variable, rate-dependent and state-dependent friction laws. Bull. Seism. Soc. Am., 92, 687-694.

Wang, J.H. (2007). A dynamic study of the frictional and viscous effects on earthquake rupture: a case study of the 1999 Chi-Chi earthquake, Taiwan. Bull. Seism. Soc. Am., 97(4), 1233-1244.

Wang, J.H. (2009). Effect of thermal pressurization on the radiation efficiency. Bull. Seism. Soc. Am., 99(4), 2293-2304.

Wang, J.H. (2011). Thermal and pore fluid pressure history on the Chelungpu fault at a depth of 1111 meters during the 1999 Chi-Chi, Taiwan, earthquake. J. Geophys. Res., 116, B03302, doi:10.1029/ 2010JB007765.

Wang, J.H. (2012). Some intrinsic properties of the two-dimensional dynamical spring-slider model of earthquake faults. Bull. Seism. Soc. Am., 102(2), 822-835.

Wang, J.H. (2013). Stability analysis of slip of a onebody spring-slider model in the presence of thermal pressurization. Ann. Geophys., 56(3), R03332, doi:10.4401/ ag-5548.

Wang, J.H. (2016a). A dynamical study of frictional effect on scaling of earthquake source displacement spectra. Ann. Geophys., 59(2), S0210, 1-14, doi:10.4401/ ag-6974.

Wang, J.H. (2016b). Slip of a one-body spring-slider model in the presence of slip-weakening friction and viscosity. Ann. Geophys. (in press)

Xu, H.J. and L. Knopoff (1994). Periodicity and chaos in a one-dimensional dynamical model of earthquakes. Phys. Rev. E, 50(5), 3577-3581.
Yoshino, (1998). Influence of crustal viscosity on earthquake energy distribution in a viscoelastic springblock system. Geophys. Res. Lett., 25, 3643-3646.
*Corresponding author: Jeen-Hwa Wang Institute of Earth Sciences, Academia Sinica, Nangang, Taipei, Taiwan; email: earth.sinica.edu.tw 2017 by Istituto Nazionale di Geofisica e Vulcanologia. All rights reserved 